ACCEPTED MANUSCRIPT

\title{
Biological and dosimetric characterisation of spatially fractionated proton minibeams
}

To cite this article before publication: Juergen Meyer et al 2017 Phys. Med. Biol. in press https://doi.org/10.1088/1361-6560/aa950C

\section{Manuscript version: Accepted Manuscript}

Accepted Manuscript is "the version of the article accepted for publication including all changes made as a result of the peer review process, and which may also include the addition to the article by IOP Publishing of a header, an article ID, a cover sheet and/or an 'Accepted

Manuscript' watermark, but excluding any other editing, typesetting or other changes made by IOP Publishing and/or its licensors"

This Accepted Manuscript is @ 2017 Institute of Physics and Engineering in Medicine.

During the embargo period (the 12 month period from the publication of the Version of Record of this article), the Accepted Manuscript is fully protected by copyright and cannot be reused or reposted elsewhere.

As the Version of Record of this article is going to be / has been published on a subscription basis, this Accepted Manuscript is available for reuse under a CC BY-NC-ND 3.0 licence after the 12 month embargo period.

After the embargo period, everyone is permitted to use copy and redistribute this article for non-commercial purposes only, provided that they adhere to all the terms of the licence https://creativecommons.org/licences/by-nc-nd/3.0

Although reasonable endeavours have been taken to obtain all necessary permissions from third parties to include their copyrighted content within this article, their full citation and copyright line may not be present in this Accepted Manuscript version. Before using any content from this article, please refer to the Version of Record on IOPscience once published for full citation and copyright details, as permissions will likely be required. All third party content is fully copyright protected, unless specifically stated otherwise in the figure caption in the Version of Record.

View the article online for updates and enhancements. 


\title{
Biological and Dosimetric Characterisation of Spatially Fractionated Proton Minibeams
}

\begin{abstract}
Juergen Meyer ${ }^{1, \xi}$, Robert D Stewart $^{1}$, Daniel Smith ${ }^{1}$, James Eagle $^{2}$, Eunsin Lee ${ }^{3,4}$, Ning Cao ${ }^{1}$, Eric Ford $^{1}$,
\end{abstract} Reza Hashemian ${ }^{1,5,6}$, Jan Schuemann ${ }^{7}$, Jatinder Saini $^{8}$, Steve Marsh $^{2}$, Rob Emery ${ }^{1}$, Eric Dorman ${ }^{1}$, Jeff Schwartz ${ }^{1}$, George Sandison ${ }^{1}$

${ }^{1}$ University of Washington, Department of Radiation Oncology, 1959 NE Pacific Street, Box 356043, Seattle, WA 98195, USA, Phone: +1 206-598-4236, Fax: + 1 206-598-6218

${ }^{2}$ University of Canterbury, Christchurch, New Zealand

${ }^{3}$ Proton Therapy Center, Cincinnati Children's Hospital, Liberty Township, OH, USA

${ }^{4}$ University of Cincinnati Medical Center, Cincinnati, OH, USA

${ }^{5}$ Christchurch District Health Board, Christchurch, New Zealand

${ }^{6}$ Cincinnati, Kenwood Radiation Oncology Center, US Oncology, Cincinnati, OH, USA

${ }^{7}$ Massachusetts General Hospital \& Harvard Medical School, Boston, MA, USA

${ }^{8}$ Seattle Cancer Care Alliance Proton Therapy Center, Seattle, WA, USA

६ Corresponding author. Email address: juergen@uw.edu

Running Title: RBE and EUD of proton minibeams 


\title{
Biological and Dosimetric Characterisation of Spatially Fractionated Proton Minibeams
}

\begin{abstract}
:
The biological effectiveness of proton beams varies with depth, spot size and lateral distance from the beam central axis. The aim of this work is to incorporate proton relative biological effectiveness (RBE) and equivalent uniform dose (EUD) considerations into comparisons of broad beam and highly modulated proton minibeams. A Monte Carlo model of a small animal proton beamline is presented. Dose and variable RBE is calculated on a per-voxel basis for a range of energies (30-109 MeV). For an open beam, the RBE values at the beam entrance ranged from 1.02-1.04, at the Bragg peak (BP) from 1.3 to 1.6, and at the distal end of the BP from 1.4 to 2.0. For a $50 \mathrm{MeV}$ proton beam, a minibeam collimator designed to produce uniform dose at the depth of the BP peak, had minimal impact on the open beam RBE values at depth. RBE changes were observed near the surface when the collimator was placed flush with the irradiated object, due to a higher neutron contribution derived from proton interactions with the collimator. For proton minibeams, the relative mean RBE weighted entrance dose (RWD) was $\sim 25 \%$ lower than the physical mean dose. A strong dependency of the EUD with fraction size was observed. For 20 Gy fractions, the EUD varied widely depending on the radiosensitivity of the cells. For radiosensitive cells, the difference was up to $\sim 50 \%$ in mean dose and $\sim 40 \%$ in mean RWD and the EUD trended towards the valley dose rather than the mean dose. For comparative studies of uniform dose with spatially fractionated proton minibeams, EUD derived from a per-voxel RWD distribution is recommended for biological assessments of reproductive cell survival and related endpoints.
\end{abstract}

Keywords: proton therapy, minibeam, variable relative biological effectiveness (RBE), linear energy transfer (LET), equivalent uniform dose (EUD), spatial modulation 


\section{Introduction}

Synchrotron-generated spatially fractionated microbeams have been reported to result in extraordinary normal tissue sparing (Dilmanian et al., 2002; Dilmanian et al., 2001; Dilmanian et al., 2003; Dilmanian et al., 2007; Bouchet et al., 2013; Bouchet et al., 2010; Serduc et al., 2008). The idea of utilizing microbeams is based on the pioneering work by Zeman and Curtis (Zeman et al., 1961; Zeman et al., 1959) at Brookhaven National Laboratory who, while conducting cosmic-ray research, discovered an inverse relationship between radiosensitivity and tissue volume exposed in mouse brain irradiated with a deuteron beam. In the same lab three decades later, Slatkin et al. (Slatkin et al., 1992) proposed microbeam radiotherapy (MRT), as a way to spare normal tissue in thin plane-parallel synchrotron beams. The dimensions of the multi-planar arrays of X-ray beams are typically in the range of 20-100 $\mu \mathrm{m}$ full width at half maximum (FWHM) with a centre-to-centre (CTC) spacing of about $100-400 \mu \mathrm{m}$ and dose rates up to the order of $10^{4} \mathrm{~Gy} / \mathrm{s}$. More recently it has been reported by Dilmanian et al. (Dilmanian et al., 2006) that wider beams up to $680 \mu \mathrm{m}$ width retain a normal tissue sparing effect, which has prompted research into alternative approaches to synchrotron-generated beams. Investigators at the Institute of Cancer Research in Sutton, UK (Bartzsch et al., 2016) proposed an MRT approach based on a conventional X-ray tube with microbeam dimensions of $50 \mu \mathrm{m}$ beam width and $400 \mu \mathrm{m}$ CTC spacing. Wider, but still submillimetre beams, are termed minibeams. Examples of X-ray based minibeam systems include the carbon nanotube based $160 \mathrm{kVp}$ system at the University of North Carolina (Zhang et al., $2014 \mathrm{~b}$ ) with a beam width of $280 \mu \mathrm{m}$. Proton based minibeam systems include the $100 \mathrm{MeV}$ beam by the group in Orsay, France (Prezado and Fois, 2013; Peucelle et al., 2015; Guardiola et al., 2017), who demonstrated the first experimental implementation in 2014; the $20 \mathrm{MeV}$ ion microprobe SNAKE by investigators in Munich (Girst et al., 2015a; Girst et al., 2016; Girst et al., 2015b; Zlobinskaya et al., 2013) who used a grid of submillimetre pencil beams, termed microchannels; and the work conducted in collaboration between Brookhaven National Laboratory and MD Anderson Cancer Center (Dilmanian et al., 2015b; Dilmanian et al., 2015a), who investigated a $109 \mathrm{MeV}$ beam with $300 \mu \mathrm{m}$ width and $1 \mathrm{~mm}$ CTC spacing. We have recently presented a feasibility study for a $50.5 \mathrm{MeV}$ proton minibeam (Lee et al., 2016a; Lee et al., 2016b) based on our image-guided precision proton radiation platform (PPRP) for preclinical in vivo research at the University of Washington (Ford et al., 2017). The multi-slit collimator was designed to produce a uniform dose at the depth of the Bragg Peak (BP), while retaining high modulation on the entrance side. The collimator dimensions are comparable to the above systems with $1 \mathrm{~mm}$ CTC spacing and $300 \mu \mathrm{m}$ slit width. 
To explore the expected biological response to this type of irradiation, and before biological experiments are conducted, exact knowledge of both the physical and biologically effective dose is crucial. It is often implicitly assumed that the RBE of low energy X-rays, as is used for synchrotron-generated microbeams, is close to unity even though a number of published studies (Nikjoo and Lindborg, 2010; Botchway et al., 1997; Fayard et al., 2002; Hoshi et al., 1988; de Lara et al., 2001; Cornforth et al., 1989; Spadinger and Palcic, 1992) indicate lower energy $(<100-200 \mathrm{kV}) \mathrm{X}$-rays are more biologically damaging per unit absorbed dose than ${ }^{60} \mathrm{Co} \gamma$-rays or MV X-rays. As an example, the RBE for DNA double strand break induction ( $\mathrm{RBE}_{\mathrm{DSB}}$ ) for a $60 \mathrm{kV}$ X-ray beam (no filtration except $0.8 \mathrm{~mm}$ Beryllium exit window) is $\sim 1.3$ (Stewart et al., 2015). It is important to note also that there is a strong correlation between $\mathrm{RBE}_{\mathrm{DSB}}$ and the $\mathrm{RBE}$ for cell survival as demonstrated recently by Streitmatter et al. (Streitmatter et al., 2017). Those investigators demonstrated that the two RBE values were within a few percent of each other for electrons and photons with energies in the range from about $250 \mathrm{eV}$ to 1 $\mathrm{MeV}$. Synchrotron-generated X-ray microbeams experiments have been reported to use median energies as low as 50-70 keV (Laissue et al., 1998; Dilmanian et al., 2002).

For clinical proton beams, Monte Carlo simulations suggest that $\mathrm{RBE}_{\mathrm{DSB}}$ may be as large as 1.2 to 1.3 near the apogee of the BP (Stewart et al., 2015). A significant difference from the purported constant (spatially invariant) RBE value of 1.1 in common clinical use (Chaudhary et al., 2014). Distal to the BP, Monte Carlo simulations (Stewart et al., 2015) suggest that $\mathrm{RBE}_{\mathrm{DSB}}$ may increase further to values as high as 1.6 to 1.8 , although only over a small distance very close to the proton range where proton fluence is relatively small compared to that incident. Increased scatter in the penumbra regions and in very small fields can also lead to higher RBE values due to energy spectrum changes (Stewart et al., 2015; Schmid et al., 2012). This is particularly relevant for very narrowly collimated, highly modulated proton beams, as path length straggling is more dominant in penumbra regions leading to lower energy protons that result in higher RBE values. Therefore, a meaningful comparison between different particle type and energy beams is only possible when RBE-weighted dose (RWD) is considered. To facilitate such comparisons, knowledge of the beam energy fluence per particle per voxel is necessary, which itself requires detailed information on the proton and secondary particle energy spectrum within the voxel.

Another factor that has complicated the comparison of experimental results derived from beams of different energy beams and/or collimator geometries is the lack of a generally accepted dose prescription and reporting 
formalism for spatially fractionated beams. For synchrotron-based spatially modulated research, the peak and valley doses as well as the peak-to-valley dose ratio (PVDR) are generally used dose parameters. For comparison with uniform beams, the dose is often specified on the beam entrance side using either Monte Carlo simulations and/or physical dosimetry approaches (Siegbahn et al., 2006; Ptaszkiewicz et al., 2008; BrauerKrisch et al., 2003; Kaplan et al., 2000; Brauer-Krisch et al., 2015), the latter approach being subject to relatively large measurement uncertainties (Crosbie et al., 2008). More recently, prescription attempts have been made that use absorbed dose values averaged over a lateral slice of material oriented perpendicular to the beam central axis for comparative studies of broad and micro- or mini-beams. For instance, Girst et al. used the mean skin dose to design an equipoised experiment to compare spatially fractionated proton pencil beams with a uniform proton beam. However, in some beam delivery cases, the Girst et al. prescription approach may not be fully appropriate. Our previous simulations of collimator-produced proton minibeams (Lee et al., 2016b) have shown that placement of a collimator relative to an irradiated object can have a considerable impact on the peak and valley doses as well as on the neutron contribution to the entrance dose (Lee et al., 2016a). This impact was also independently verified by Guardiola et al (Guardiola et al., 2017). So even if the mean physical dose between modulated and uniformly irradiated scenarios is equivalent, the RWD may not be biologically equivalent, especially when proton minibeams are compared to microbeams or broad beams of $\mathrm{kV}$ or MV Xrays.

Explicit considerations of variable RBE and spatial variations in physical dose must be taken into consideration when designing experiments to probe for new or refined biological mechanisms of action underlying the potential therapeutic advantages of micro- or mini-beam irradiation. In most publications related to micro- and minibeam experiments produced by collimation, it is not clear if the exact positioning and orientation of the collimator was carefully considered in the dosimetry or if beam spectral changes were considered with beam line positioning changes and possibly other components. Of note is one innovative experimental approach to determine the dose equivalence between microbeam irradiation and conventional broad beams. Ibahim et al. (Ibahim et al., 2014) used clonogenic and cell impedance assays, but highlighted that their dosimetry of the synchrotron beam was based on an idealized geometry and it was therefore likely that the valley dose was underestimated. Another approach was proposed by Zhang et al. for clinical X-Ray based grid therapy for melanoma on a linear accelerator (Zhang et al., 2014a). They used the Linear-Quadratic (LQ) model and calculated the equivalent uniform dose (EUD). 


\section{Methods}

The University of Washington (UW) research proton beam line was modelled by means of Monte Carlo (MC) simulations in TOPAS (Perl et al., 2012), version 3.0.p1. Compared to previous work by our group (Lee et al., 2016b; Ford et al., 2017), modifications have been made to the proton beamline to integrate it with the isocenter of a Small Animal Radiation Research Platform (SARRP) (XStrahl Ltd, Surrey, UK). The changes affected the beam characteristics and therefore several components had to be added or moved when depicted in the simulations. The UW cyclotron produces a $50.5 \mathrm{MeV}$ monoenergetic proton beam before it enters the research beamline. The components modelled in the beamline include 1) a $1.23 \mathrm{~mm}$ thick graphite beam degrader upstream, 2) a "large" stainless steel beam pipe, 3) a graphite/stainless steel stray beam detector that connects the large beam pipe with 4) a smaller stainless-steel beam pipe with inner diameter $35 \mathrm{~mm}$ including 5) a 0.15 mm thick Kapton exit window to contain the vacuum and 6) a protruding small beam pipe downstream of the Kapton that is open to air. Between the beam exit window and a water phantom with a $1 \mathrm{~mm}$ Lexan entrance window is a large Bragg peak ionization chamber (Model T34080, PTW, Freiburg, Germany), henceforth 
referred to as the monitoring ion chamber. The monitoring ion chamber is necessary to trigger and terminate the beam. A multi-slit minibeam collimator can be placed between the monitoring ion chamber and the water phantom. A schematic of the beamline from the stray beam detector to the water phantom, but without the monitoring chamber, can be found in Lee et al. as Figure 1 (Lee et al., 2016b). A prototype multi-slit collimator was made from $25 \mathrm{~mm}$ thick SAE 304 steel. It contained thirty-one $300 \mu \mathrm{m}$ wide slits with a CTC spacing of 1 $\mathrm{mm}$ covering a field size of $3 \times 3 \mathrm{~cm}$. The multi-slit collimator was designed to produce a uniform dose at the depth of the BP via post collimator scattering in water while retaining the produced high spatial modulation at shallow depths in the water.

The exact positioning of the monitoring ion chamber and the water phantom was modelled in TOPAS. Simulations were run using $10^{9}$ proton particle histories. The TOPAS simulations were run on two processors (Intel(R) Xeon(R) CPU E5-2697 v3 @ 2.60GHz, with 14 cores each, 256 GB RAM). The voxel dimension along the beam axis for simulation computations were $0.05 \mathrm{~mm}$, and $0.8 \mathrm{~mm}$ and $0.05 \mathrm{~mm}$ along and across the direction of the modulation of the beam, respectively. Binary output files were generated and imported into MATLAB (MathWorks, Natick, MA, USA) for further analysis.

\subsection{Physical dose}

To investigate and verify the beam energy, the first step was to validate the modified beamline model in TOPAS without the multi-slit collimator in place. Modelling the beam energy correctly is the basis for accurate modelling of the LET and RBE. Percentage depth dose (PDD) measurements were conducted with an Exradin Spokas A11 parallel plate ion chamber with a collecting volume of $0.62 \mathrm{cc}$ (Standard Imaging, Middleton, WI, USA) and a microDiamond detector (Type 60019, PTW-Freiburg, Germany) with a nominal sensitive volume of $0.004 \mathrm{~mm}^{3}$. The A11 ion chamber was manually positioned along the central axis by means of a micrometre motion stage in $100 \mu \mathrm{m}$ steps around the BP and $1 \mathrm{~mm}$ in steps in the build-up region. The microDiamond detector was positioned by means of a motion stage (Saini et al., 2017) every $500 \mu \mathrm{m}$.

\subsection{LET- and RWD for the endpoint of DSB Induction}

The dose-averaged LET, averaged over all protons and secondary particles, was calculated by TOPAS on a pervoxel basis. Dose-averaged LET is slightly larger than the track-averaged LET (Granville and Sawakuchi, 2015; Guan et al., 2015). The RBE $\mathrm{DSB}_{\mathrm{DB}}$ within the voxel was simulated using information from the Monte Carlo 
Damage Simulation (MCDS) (Stewart et al., 2015; Stewart et al., 2011) as implemented in TOPAS (Polster et al., 2015). Estimates of $\mathrm{RBE}_{\mathrm{DSB}}$, which have been extensively benchmarked against measured data and track structure simulations (Stewart et al., 2011; Streitmatter et al., 2017), are based on ${ }^{60} \mathrm{Co} \gamma$-rays as reference radiation (Stewart et al., 2015). For electrons and photons, $\mathrm{RBE}_{\mathrm{DSB}}$ is within a few percent of the RBE for reproductive cell survival (Streitmatter et al., 2017). For Z $>1$ particles, the repair-misrepair-fixation (RMF) model (Carlson et al., 2008) predicts that the RBE for cell survival will be greater than or equal to RBE $E_{D S B}$. Thus, the $\mathrm{RBE}_{\mathrm{DSB}}$ values presented in this work represent the lower bound on the RBE for cell survival for comparison to a constant RBE of 1.1. The RWD was computed on a per-voxel basis for both the open and spatially modulated beams with the collimator placed at different distances from the water phantom.

\subsection{Calculation of Equivalent Uniform Dose}

The equivalent uniform dose is defined as "the uniform dose that, if delivered over the same number of fractions as the non-uniform dose distribution of interest, yields the same radiobiological effect" (AAPM Task Group 166, 2012). The EUD (Niemierko, 1997) was applied to evaluate and compare uniform with non-uniform irradiation geometries. For the endpoint of cell survival, the EUD is given by

$$
E U D=\frac{\alpha / \beta}{2}\left(-1+\sqrt{1-\frac{4 \ln \bar{S}}{\alpha(\alpha / \beta)}}\right), \text { where } \bar{S}=\frac{1}{V} \sum_{i=1}^{n} v_{i} \exp \left(-\alpha D_{i}-\beta D_{i}{ }^{2}\right)
$$

$\alpha$ and $\beta$ are parameters in the LQ cell survival model, $\bar{S}$ is the surviving fraction (SF), $v_{i}$ is the volume of a single voxel, $V=\sum_{i=1}^{n} v_{i}$ is the total volume of the region of interest (ROI), and $n$ is the number of voxels in the ROI. In this work, the concept of EUD is explored with both the physical dose, $D$, and the RBE-weighted dose, $R W D$ (instead of $D$ ). $R W D_{i}$ is the ${ }^{60} \mathrm{Co} \gamma$-ray absorbed dose delivered to the $i^{\text {th }}$ voxel that creates the same number of DSB per cell as proton absorbed dose $D_{i} \times R B E_{i}$. For the special case when $v_{i}=v=V / n$, the surviving fraction averaged over all $n$ voxels computed using RWD is

$$
\bar{S}=\frac{1}{n} \sum_{i=1}^{n} \exp \left(-\alpha R W D_{i}-\beta R W D_{i}{ }^{2}\right)=\frac{1}{n} \sum_{i=1}^{n} \exp \left[-\alpha R W D_{i}\left(1+\frac{R W D_{i}}{(\alpha / \beta)}\right)\right] .
$$




$$
\bar{S}=\frac{1}{n} \sum_{i=1}^{n} \exp \left(-\alpha R W D_{i}\right)
$$

For the sake of this comparison, we do not distinguish between tumour cells and normal tissues per se but investigate the concept more generally for a representative range of $\alpha / \beta$ ratios ( 1 and $10 \mathrm{~Gy})$. To bracket the range of radiosensitive to radioresistant tissues, we assume surviving fractions between 0.1 and 0.95 , respectively, after uniform irradiation by a 2 Gy proton dose at the BP. The EUD formalism was calculated in MATLAB and applied to the imported physical and dose-averaged RBE from TOPAS. Both the mean doses and the EUD were computed along the central axis $(\mathrm{CAX})$ across each $0.05 \mathrm{~mm}$ voxel layer within the $3 \times 3 \mathrm{~cm}$ field size.

\section{Results}

Figure 1 shows the energy spectrum at various stages along the beam delivery system of the initially monoenergetic $50.5 \mathrm{MeV}$ proton beam emerging from the cyclotron. The fluence after the degrader is reduced by $>90 \%$. The aim of the degrader is to produce a uniform beam at the end of the beam pipe. Both the degrader as well as the monitoring ion chamber reduce the peak energy by about 2-3 MeV resulting in a dominant peak energy of $45 \mathrm{MeV}$ after the monitoring ion chamber with a narrow energy spread of $\pm 0.5 \mathrm{MeV}$. There is a tail of proton energies between 25 and $42 \mathrm{MeV}$ with a maximum of $0.9 \%$ of the counts at the peak energy (see proton energy histogram in Figure 2a). The tail primarily stems from particles scattered off the beam pipe wall. In Figures $2 b-d$, the effect of the minibeam collimator on the proton energy spectrum is shown (secondary axis) when placed flush and at 2 and $5 \mathrm{~cm}$ distant from the water phantom, respectively. Also shown in Figure 2 (primary axis) is the corresponding relative neutron fluence. The relative magnitude of the maximum neutron fluence is less than $0.8 \%$ with the collimator flush and $0.3 \%$ and $0.2 \%$ when placed at 2 and $5 \mathrm{~cm}$ distant from the surface, respectively.

For the measured PDDs for the open beam, the depth of $17.0 \mathrm{~mm}$ for the BP agrees well with the simulations (Figure 3). The plots were normalized to $1 \mathrm{~mm}$ depth. The depth of the BP corresponds to the beam's most probable incident energy and the sharpness of the BP corresponds to the degree of energy distribution spread in the beam about the BP depth. The agreement between the modelled and measured depth-dose curves validates that the Monte Carlo model adequately represents the UW proton beam line. The Bragg-peak-to-entrance-ratios (BPER) were 4.8:1, 4.3:1 and 3.9:1 for the simulated beam, the ion chamber measurement and the 
microdiamond measurement, respectively. The difference in the peak dose at the BP reflects the different resolution of the simulated and measured data and the resulting volume averaging effect in the detectors used.

A comprehensive analysis of the open beam PDD (dose) compared to the RWD based on both a constant RBE of 1.1 (cRWD) and the computed variable $\mathrm{RBE}_{\mathrm{DSB}}(\mathrm{vRWD})$ are shown in Figure 4. The graphs illustrate the RBE effects of the proton beam in the absence of a collimator. The LET, a constant RBE of 1.1 (cRBE) and the $\mathrm{RBE}_{\mathrm{DSB}}(\mathrm{vRBE})$ are plotted in Figure $4 \mathrm{~b}$. The relative differences in RBE and the absolute differences in RWD are shown in Figure $4 \mathrm{c}$ and $4 \mathrm{~d}$, respectively. LET values for the beam at depth were $2.7 \mathrm{keV} / \mu \mathrm{m}$ at $10 \mathrm{~mm}$, and $4.8 \mathrm{keV} / \mu \mathrm{m}$ at $15 \mathrm{~mm}$. LET increased near-linearly to $15.1 \mathrm{keV} / \mu \mathrm{m}$ at the $\mathrm{BP}$ and reached a maximum value of $30.1 \mathrm{keV} / \mu \mathrm{m}$ at the distal end of the BP. A similar behaviour with depth was exhibited for $\mathrm{RBE}_{\mathrm{DSB}}$ with predicted values of $1.05,1.1,1.4$ and 1.9 , corresponding to the above depths, respectively.

For comparison, we also computed $\mathrm{RBE}_{\mathrm{DSB}}$ for a range of energies on our beamline (without multi-slit collimator) to examine the energy dependence and magnitude of the $\mathrm{RBE}_{\mathrm{DSB}}$ over the range of available energies at other proton minibeam facilities. The results are given in Table 1. The expected penetration in water of a nominally $20 \mathrm{MeV}$ beam is approximately $4 \mathrm{~mm}$. However, no meaningful data could be obtained with our beam components, given the attenuation along the UW beamline at such a low energy, and therefore no values are included. LET and $\mathrm{RBE}_{\mathrm{DSB}}$ values comparing the non-collimated (open) beam with the collimator at different distances from the phantom are shown in Table 2.

The RBE effects on the dose profiles are shown in Figure 5. Figures 5a-b show the dose and the RWD at the phantom surface and at the depth of the BP with the collimator placed flush with the water phantom, respectively. In Figures 5e-f, the corresponding information is displayed for the collimator placed at $2 \mathrm{~cm}$ from the water phantom. Figures 5c-d and 5g-h show the relative difference between the dose and RWD, respectively.

The PDDs through the central peak and valley for the collimated beam are shown in Figure 6. A comparison of the mean dose and EUD range for different $\alpha / \beta$ ratios and surviving fractions is also shown for two scenarios. Figures $6 \mathrm{a}$ and $6 \mathrm{~b}$ correspond to the collimator flush with the water phantom and Figures $6 \mathrm{c}$ and $6 \mathrm{~d}$ show the collimator placed at $2 \mathrm{~cm}$ distance. Figures $6 \mathrm{a}$ and $6 \mathrm{c}$ refer to the physical absorbed dose and Figures $6 \mathrm{~b}$ and $6 \mathrm{~d}$ to the RWD along the central axis. The graphs are normalised to $2 \mathrm{~Gy}$ at central axis (peak). Figures $6 \mathrm{e}$ and $6 \mathrm{f}$ 1 


\section{Discussion}

Determining the biologically equivalent dose deposited by a polyenergetic proton beam requires exact knowledge of the beam's spectrum as a function of spatial location in the absorbing medium. To gain this knowledge, we developed a Monte Carlo simulation model of the University of Washington PPRP beamline including all of its pertinent components. The beam energy of this model was verified by means of PDD measurements (Figure 3) and a comprehensive analysis was carried out with respect to the influence of the most relevant components of the beam spectrum (Figure 1 and 2a). Although the beam is degraded by about $5 \mathrm{MeV}$ along the beam path, to a peak energy of $45 \mathrm{MeV}$, the initially monoenergetic proton beam remains mostly pristine with only a small energy spread of $\pm 0.5 \mathrm{MeV}$. This is important as the sharpness of the BP is greatest for a monoenergetic beam and deteriorates when the proton energy spectrum broadens. To generate spatially modulated beams, the beam spot can either be scanned (Klodowska et al., 2015) or generated by a multi-slit collimator (Lee et al., 2016b). The effects of a multi-slit collimator for proton minibeams at different distances from a phantom were modelled and the results are shown in Figure 2. The collimator does not further degrade the proton energies within the beam; rather, it results in a slight narrowing of the $45 \mathrm{MeV}$ peak and reduces the tail of scattered energies between 35 and $42 \mathrm{MeV}$ regardless of its position relative to the phantom. However, the neutron contribution increased when the collimator was introduced, especially when the collimator was placed flush with the phantom surface, leading to an increase by approximately a factor of 8 (Figure 2b). Overall, the neutron contribution is very small, less than $1 \%$ of the proton counts, but due to the higher RBE of neutrons, and subsequently higher RWD, the relative biologically relevant dose contribution is higher. The neutron contribution at the phantom surface reduces sharply as a function of distance of the collimator from the phantom surface and is almost equivalent to that of a non-collimated beam when placed $5 \mathrm{~cm}$ distant from the surface. These observations are in line with our previous findings (Lee et al., 2016a) and those independently made by Guardiola et al. (Guardiola et al., 2017). Thus, to decrease the number of neutrons on the surface, the collimator should be placed away from the entrance. Unfortunately, this also will decrease the collimator's effective degree of spatial modulation, i.e. the PVDR. For the current collimator simulations, the PVDR at the entrance was 37 when the collimator was placed flush (Figure 6a) and reduced by a factor of 10 to 3.7 at $2 \mathrm{~cm}$ distant from the surface (Figure 6c). This further highlights the strong dependency of the dosimetry of these 
spatially modulated proton beams on the position of the collimator relative to the irradiated object, as previously described (Lee et al., 2016b; Guardiola et al., 2017).

\section{RBE considerations}

Although DSB are widely considered one of the more biologically challenging forms of DNA damage to repair, the majority $(>95-97 \%$ ) of the initial DSB formed by ionizing radiation are correctly rejoined. The RepairMisrepair-Fixation (RMF) model (Mairani et al., 2016; Carlson et al., 2008; Frese et al., 2012; Streitmatter et al., 2017) explicitly links the formation and subsequent biological processing of initial DSB to the formation of lethal and non-lethal chromosome aberrations. In the RMF model, the RBE for cell survival in the limit as the dose becomes large compared to $(\alpha / \beta)$ is exactly equal to $\mathrm{RBE}_{\mathrm{DSB}}$. For doses comparable to or smaller than $(\alpha / \beta)$, the interactions of pairs of DSB formed by the same track (intra-track binary misrepair) are more likely to form lethal damage than pairs of DSB formed by different tracks (inter-track binary misrepair). These proximity effects (Hlatky et al., 2002) imply that the low dose RBE for cell survival $\left(R B E_{L D}\right)$ is greater than the high dose $\operatorname{RBE}\left(R B E_{H D}\right)$ for cell survival. For protons, Carabe-Fernandez et al. (Carabe-Fernandez et al., 2007; CarabeFernandez et al., 2010) also found that the RBE for cell survival decreases with increasing dose, i.e., $R B E_{\max }=$ $R B E_{L D}$ and $R B E_{\text {min }}=R B E_{H D}$. For proton mini-beams, it is reasonable to expect the RBE for cell survival to approach $R B E_{\text {min }}=R B E_{H D}=R B E_{D S B}$ in regions of tissue in which the peak or valley dose is comparable to or exceeds $(\alpha / \beta)$. Additional work is needed to examine RBE effects in spatially modulated particle beams in the limit of valley or peak doses that are small compared to $(\alpha / \beta)$.

The relative contribution of the different particles and energies in the beam have an effect on the spatial distribution of RWD. The implementation of the MCDS model of RBE $\mathrm{DSB}_{\mathrm{B}}$ into TOPAS allows this dependency to be modelled on a per-voxel basis. Before investigating the spatially modulated beam, the LET and RBE ${ }_{\mathrm{DSB}}$ distribution along the central axis were examined for an open beam to serve as a reference. For comparison with other facilities that have a proton minibeam, simulations were run for energies published in the range 30 to 109 $\mathrm{MeV}$. To reiterate, the values provided in Table 1 reflect the nominal beam energies of the mono-energetic proton beam before entering the beam pipe on the UW beam line. The beam energy incident at the phantom surface is therefore lower and might be different if injected into a beamline at another facility. The purpose of the comparison was to get a sense of the approximate differences in biologically equivalent dose between different facilities where investigators are exploring spatially fractionated proton beams. This is important when 
comparing results generated by different beamlines. The values show that, as expected, both LET and RBEDSB decrease with increasing beam energy. However, for the $50.5 \mathrm{MeV}$ beam and higher energies, the RBE $\mathrm{DSB}_{\text {at }} 1$ $\mathrm{mm}$ depth is nearly identical $(1.03 \pm 0.01)$; the same is true at the BP, where the RBE is $1.33 \pm 0.08$. The $\mathrm{RBE}_{\mathrm{DSB}}$ towards the distal end of the BP shows higher variation, but due to the lower physical dose delivered, this typically has less of an impact. For lower energies, the RBE ${ }_{D S B}$ increases more drastically. Overall, the $\mathrm{RBE}_{\mathrm{DSB}}$ values between the surface and depth of the BP are, while continuously changing, comparable for nominal energies between 50-109 MeV. For lower energy beams not included here, higher LET and RBE $\mathrm{DSB}_{\mathrm{D}}$ values are expected.

For the UW $50.5 \mathrm{MeV}$ beam, both LET and $\mathrm{RBE}_{\mathrm{DSB}}$ follow a similar trend with noisier values towards the distal end of the BP (Figure 4). The RWD is close in value to the relative physical dose between the surface and the proximal side of the BP. The steep near-linear increase in $\mathrm{RBE}_{\mathrm{DSB}}$ starts at about $1 \mathrm{~mm}$ before and ends $1 \mathrm{~mm}$ after the BP. For clinical beams at higher energies, a constant RBE of 1,1, referred to as cRBE, is typically used. It is shown in Figure 4 that along the CAX a constant RBE of 1.1 overestimates $\mathrm{RBE}_{\mathrm{DSB}}$ by approximately $5 \%$ down to a depth of $12 \mathrm{~mm}$, then the two are about equal for a few/mm before $\mathrm{RBE}_{\mathrm{DSB}}$ increases sharply. The near-linear trend in the LET and RBE $E_{\mathrm{DSB}}$ towards the end of the beam is not reflected in the RWD difference between the vRWD and the cRWD (Figure 4d). Effectively the largest RWD difference is past the BP at a depth of $17.15 \mathrm{~mm}$ and tapers off to either side.

Table 2 shows that placing a collimator in the beam path does not substantially affect the LET and RBE values at the BP. The effects of the RWD for a modulated beam generated with a steel collimator are shown in Figures 5 and 6. Note that Figure 5 is normalized to the CAX at $1 \mathrm{~mm}$ depth and Figure 6 to the CAX at the depth of the BP. At shallow depths, the valleys and peaks are about $5 \%$ lower than the constant RBE value of 1.1 that is commonly clinically assumed (Figure $5 \mathrm{c}$ and $5 \mathrm{~g}$ ). While this does not affect the PVDR considerably, it does lead to lower valley doses, which have been reported to correspond to the normal tissue tolerance of uniformly irradiated beams (Siegbahn et al., 2006). At a depth around the BP, the higher RWD result in higher peak doses by a factor of $43 \%$ at the BP (Figures $5 \mathrm{~d}$ and $\mathrm{h}$ ). When the RWD is normalized to the BP, as was done for Figure 6, the relative entrance valley doses are therefore reduced. Also of note are the increased $\mathrm{RBE}_{\mathrm{DSB}}$ yalues in the penumbra regions resulting in an increased RWD. This can be seen in Figures $5 c-d$ and $5 \mathrm{~g}-\mathrm{h}$. While the physical dose between the collimator placed flush with the phantom (Figure $5 \mathrm{a}-\mathrm{b}$ ) and at $2 \mathrm{~cm}$ 
distant (Figure 5e-f) are quite different, especially in the valley regions, the relative differences are identical (compare Figures 5c-d with Figures 5g-h) indicating that no noticeable RBE ${ }_{D S B}$ changes take place in the penumbra for the two different collimator positions. This is because the collimator produced minibeam energy spectrum in the penumbra is essentially unaffected by the additional small amount of scattering caused by $2 \mathrm{~cm}$ of air.

\section{EUD considerations}

Zeman and Curtis (Zeman et al., 1961; Zeman et al., 1959) observed the radiosensitivity of small tissue volumes was dramatically decreased when irradiated by microbeams of X-rays compared to uniform beam irradiation. A satisfactory explanation of this phenomenon remains elusive. However, separating the contribution of known radiation response observation influence factors such as RBE and EUD between the two very different radiation delivery approaches is an important step toward other identifying possible explanations for the overall phenomenon. Although the EUD concept lends itself well to characterizenon-uniform 3D dose distributions, it is not without limitations and may not fully account for all radiation biological response factors. The LQ model is inadequate to capture all dose-response characteristic factors for tumour or tissue, such as e.g. re-oxygenation, repopulation, re-distribution of cells in cell cycle, vascular damage and immune response. The LQ model only corrects for repair and may or may not be sufficiently accurate as the dose per fraction becomes large compared to $\alpha / \beta$ (Brown et al., 2014; Kirkpatrick et al., 2008; Brenner, 2008). It has also recently been reported that the LQ model may not be adequate to predict the response for spatially fractionated fields as it does not sufficiently account for bystander effects (Peng et al., 2017). Consequently, the above plus other unknown biological processes that possibly contribute to the overall response of the cells within or near a tissue region of interest may not be captured by EUD when radiation response is elicited by highly spatially fractionated beams. With this in mind, when comparing uniform with spatially fractionated dose distributions it is therefore imperative to compute the EUD to be able to separate known from unknown factors. For synchrotron based microbeam radiotherapy, decreased radiosensitivity effects have commonly been referred to as the "normal tissue sparing effect" (Dilmanian et al., 2007).

To investigate the influence of the EUD on different sensitivity cells and as a function of dose (c.f. Eq. $1 \& 2$ ), the EUD was calculated for $2 \mathrm{~Gy}$ and $20 \mathrm{~Gy}$ at the BP for a range of surviving fractions ( 0.1 to 0.95$)$ and $\alpha / \beta$ ratios (1 and $10 \mathrm{~Gy})$ for the UW proton mini beam. This EUD dependency is shown together with the mean 
dose in the plot of the PDDs in the peak and valley regions in Figure 6. Figure 6c-d show that the EUD is close to the mean physical dose and the mean RWD but not identical when the collimator is $2 \mathrm{~cm}$ distant. The larger difference is seen for the collimator placed flush (Figure 6a-b). While the overall difference between the mean dose and EUD is indistinguishable in the BP region, the difference is most obvious on the beam entrance side down to about $10 \mathrm{~mm}$ depth. The higher neutron contribution with the collimator placed flush (Figure 2a) is the main contributing factor for this observation. Therefore, the BP dose is relatively uniform and the physical dose, the EUD and mean dose are very similar per their definition. For the higher $\alpha / \beta$ ratios, the EUD tends to be lower than the mean dose, whereas for the lower $\alpha / \beta$ ratios the EUD tends to be higher. In terms of the peak and valley doses, the difference in the shape of the PDDs between the different collimator positions is substantial. In Fig. 6c-d, the relative peak dose along the collimator slit is much lower and remains nearly constant with depth down to the proximal end of the BP, whereas the relative valley dose increases somewhat. Despite the differences in the PVDR between the collimator placed flush and $2 \mathrm{~cm}$ distant, the mean dose and the mean RWD are nearly identical as pointed out above. Increasing the dose to 20 Gy thus does not affect the relative mean dose. However, since the EUD is dependent on dose, large deviations between the EUD and the mean dose can occur. For larger doses, the EUD tends towards the valley dose, especially for the radiosensitive cells $(\mathrm{SF}=0.1)$ regardless of $\alpha / \beta$ ratio. It can be clearly seen that for large doses the mean dose and mean RWD overestimate the EUD. Therefore, the mean dose and mean RWD are not adequate metrics to compare uniform and non-uniform proton beams for the endpoint of reproductive cell survival.

This work primarily investigates proton minibeams but is also relevant more generally for X-Ray based spatially modulated microbeams for which cell and small animal data are available. For example, early small animal experiments that reported extraordinary normal tissue sparing and a greater therapeutic index of modulated synchrotron microbeams used median X-ray energies in the range 50-70 keV (Laissue et al., 1998; Dilmanian et al., 2002) with peak skin entrance doses as high as 500 Gy. RBE and EUD considerations would most likely affect the reported results, such as e.g. a reported gain in therapeutic index of a factor of $\sim 5$ (Dilmanian et al., 2002) for microbeam therapy over uniform irradiation. This is not to say that bystander effects have no additional significance for modulated beams, which in fact has been demonstrated consistently. On the contrary, accounting for the biological dose equivalence of modulated and uniformly irradiated beams enables better experimental design and reporting of the aforementioned effects. The experimental verification of the models and considerations presented in this work should be the next step and remains an area of further work. 


\section{Conclusion}

Proton minibeams in the energy range $20-109 \mathrm{MeV}$ exhibit relatively large RBE variations, especially around the BP and the penumbra regions. These variations can be modelled with Monte Carlo simulations and should be reported for dosimetric and experimental studies. For comparison of spatially fractionated and uniform dose distributions the EUD can considerably deviate from the mean dose, especially for high radiation doses and radiosensitive tumours. Both RBE and EUD corrections are strongly recommended when designing and reporting about comparative studies between uniform and highly spatially fractionated dose distributions. This will facilitate fair comparisons between different type beams and interinstitutional experimental setups as well as better quantification of the effects that may not be adequately described with the LQ model.

\section{Acknowledgements}

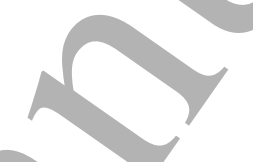

This research was supported in part by a University of Washington Royalty Research Fund under Grant No.

A116434. 
References

AAPM Task Group 1662012 The Use and QA of Biologically Related Models for Treatment Planning. ed American Association of Physicists in Medicine (One Physics Ellipse, College Park, MD 20740-3846

Bartzsch S, Cummings C, Eismann S and Oelfke U 2016 A preclinical microbeam facility with a conventional x-ray tube Medical physics 436301

Botchway S W, Stevens D L, Hill M A, Jenner T J and O'Neill P 1997 Induction and rejoining of DNA double-strand breaks in Chinese hamster V79-4 cells irradiated with characteristic aluminum $\mathrm{K}$ and copper $\mathrm{L}$ ultrasoft $\mathrm{X}$ rays Radiation research 148 317-24

Bouchet A, Lemasson B, Christen T, Potez M, Rome C, Coquery N, Le Clec'h C, Moisan A, Brauer-Krisch E, Leduc G, Remy C, Laissue J A, Barbier E L, Brun E and Serduc R 2013 Synchrotron microbeam radiation therapy induces hypoxia in intracerebral gliosarcoma but not in the normal brain Radiotherapy and oncology 108 143-8

Bouchet A, Lemasson B, Le Duc G, Maisin C, Brauer-Krisch E, Siegbahn E A, Renaud L, Khalil E, Remy C, Poillot C, Bravin A, Laissue J A, Barbier E L and Serduc R 2010 Preferential effect of synchrotron microbeam radiation therapy on intracerebral 9L gliosarcoma vascular networks International journal of radiation oncology, biology, physics 78 1503-12

Brauer-Krisch E, Adam J F, Alagoz E, Bartzsch S, Crosbie J, DeWagter C, Dipuglia A, Donzelli M, Doran S, Fournier P, Kâlef-Ezra J, Kock A, Lerch M, McErlean C, Oelfke U, Olko P, Petasecca M, Povoli M, Rosenfeld A, Siegbahn E A, Sporea D and Stugu B 2015 Medical physics aspects of the synchrotron radiation therapies: Microbeam radiation therapy (MRT) and synchrotron stereotactic radiotherapy (SSRT) Physica medica : PM : an international journal devoted to the applications of physics to medicine and biology : official journal of the Italian Association of Biomedical Physics 31 568-83

Brauer-Krisch E, Bravin A, Lerch M, Rosenfeld A, Stepanek J, Di Michiel M and Laissue J A 2003 MOSFET dosimetry for microbeam radiation therapy at the European Synchrotron Radiation Facility Medical physics 30 583-9

Brenner D J 2008 The linear-quadratic model is an appropriate methodology for determining isoeffective doses at large doses per fraction Semin Radiat Oncol 18 234-9

Brown J M, Carlson D J and Brenner D J 2014 The Tumor Radiobiology of SRS and SBRT: Are More Than the 5 Rs Involved? Int J Radiat Oncol 88 254-62

Carabe-Fernandez A, Dale R G, Hopewell J W, Jones B and Paganetti H 2010 Fractionation effects in particle radiotherapy: implications for hypo-fractionation regimes Physics in medicine and biology 55 5685-700

Carabe-Fernandez A, Dale R G and Jones B 2007 The incorporation of the concept of minimum RBE (RbEmin) into the linear-quadratic model and the potential for improved radiobiological analysis of high-LET treatments International journal of radiation biology $\mathbf{8 3} 27-39$

Carlson D J, Stewart R D, Semenenko V A and Sandison G A 2008 Combined use of Monte Carlo DNA damage Simulations and deterministic repair models to examine putative mechanisms of cell killing Radiation research 169 447-59

Chaudhary P, Marshall T I, Perozziello F M, Manti L, Currell F J, Hanton F, McMahon S J, Kavanagh J N, Cirrone G A P, Romano F, Prise K M and Schettino G 2014 Relative Biological Effectiveness Variation Along Monoenergetic and Modulated Bragg Peaks of a 62-MeV Therapeutic Proton Beam: A Preclinical Assessment Int J Radiat Oncol $9027-35$ 
Cornforth M N, Schillaci M E, Goodhead D T, Carpenter S G, Wilder M E, Sebring R J and Raju M R 1989 Radiobiology of ultrasoft X rays. III. Normal human fibroblasts and the significance of terminal track structure in cell inactivation Radiation research 119 511-22

Crosbie J C, Svalbe I, Midgley S M, Yagi N, Rogers P A and Lewis R A 2008 A method of dosimetry for synchrotron microbeam radiation therapy using radiochromic films of different sensitivity Physics in medicine and biology 53 6861-77

de Lara C M, Hill M A, Jenner T J, Papworth D and O'Neill P 2001 Dependence of the yield of DNA double-strand breaks in Chinese hamster V79-4 cells on the photon energy of ultrasoft X rays Radiation research 155 440-8

Dilmanian F, Zhong Z, Bacarian T, Benveniste H, Romanelli P, Wang R, Welwart J, Yuasa T, Rosen E M and Anschel D J 2006 Interlaced x-ray microplanar beams: a radiosurgery approach with clinical potential Proc Natl Acad Sci U S A 103 9709-14

Dilmanian F A, Button T M, Le Duc G, Zhong N, Pena L A, Smith J A, Martinez S R, Bacarian T, Tammam J, Ren B, Farmer P M, Kalef-Ezra J, Micca P L, Nawrocky M M, Niederer J A, Recksiek F P, Fuchs A and Rosen E M 2002 Response of rat intracranial 9L gliosarcoma to microbeam radiation therapy Neuro Oncol 4 26-38

Dilmanian F A, Eley J G and Krishnan S 2015a Minibeam therapy with protons and light ions: physical feasibility and potential to reduce radiation side effects and to facilitate hypofractionation International journal of radiation oncology, biology, physics 92 469-74

Dilmanian F A, Eley J G, Rusek A and Krishnan S 2015b Charged Particle Therapy with Mini-Segmented Beams Frontiers in oncology 5269

Dilmanian F A, Morris G M, Le Duc G, Huang X, Ren B, Bacarian T, Allen J C, Kalef-Ezra J, Orion I, Rosen E M, Sandhu T, Sathe P, Wu X Y, Zhong Z and Shivaprasad H L 2001 Response of avian embryonic brain to spatially segmented x-ray microbeams Cell Mol Biol (Noisy-le-grand) 47 485-93

Dilmanian F A, Morris G M, Zhong N, Bacarian T, Hainfeld J F, Kalef-Ezra J, Brewington L J, Tammam J and Rosen E M 2003 Murine EMT-6 carcinoma: high therapeutic efficacy of microbeam radiation therapy Radiation research $159632-41$

Dilmanian F A, Qu Y, Feinendegen L E, Pena L A, Bacarian T, Henn F A, Kalef-Ezra J, Liu S, Zhong Z and McDonald J W 2007 Tissue-sparing effect of x-ray microplanar beams particularly in the CNS: is a bystander effect involved? Exp Hematol 35 69-77

Fayard B, Touati A, Abel F, Herve du Penhoat M A, Despiney-Bailly I, Gobert F, Ricoul M, L'Hoir A, Politis M F, Hill M A, Stevens D L, Sabatier L, Sage E, Goodhead D T and Chetioui A 2002 Cell inactivation and double-strand breaks: the role of core ionizations, as probed by ultrasoft $\mathrm{X}$ rays Radiation research 157 128-40

Ford E, Emery R, Huff D, Narayanan M, Schwartz J, Cao N, Meyer J, Rengan R, Zeng J, Sandison G, Laramore G and Mayr N 2017 An image-guided precision proton radiation platform for preclinical in vivo research Physics in medicine and biology $\mathbf{6 2}$ 43-58

Frese M C, Yu V K, Stewart R D and Carlson D J 2012 A mechanism-based approach to predict the relative biological effectiveness of protons and carbon ions in radiation therapy International journal of radiation oncology, biology, physics $\mathbf{8 3} 442-50$

Girst S, Greubel C, Reindl J, Siebenwirth C, Zlobinskaya O, Dollinger G and Schmid T E 2015a The influence of the channel size on the reduction of side effects in microchannel proton therapy Radiation and environmental biophysics $\mathbf{5 4} 335-42$

Girst S, Greubel C, Reindl J, Siebenwirth C, Zlobinskaya O, Walsh D W, Ilicic K, Aichler M, Walch A, Wilkens J J, Multhoff G, Dollinger G and Schmid T E 2016 Proton 
Minibeam Radiation Therapy Reduces Side Effects in an In Vivo Mouse Ear Model International journal of radiation oncology, biology, physics 95 234-41

Girst S, Marx C, Brauer-Krisch E, Bravin A, Bartzsch S, Oelfke U, Greubel C, Reindl J, Siebenwirth C, Zlobinskaya O, Multhoff G, Dollinger G, Schmid T E and Wilkens J J $2015 \mathrm{~b}$ Improved normal tissue protection by proton and X-ray microchannels compared to homogeneous field irradiation Physica medica : PM : an international journal devoted to the applications of physics to medicine and biology :official journal of the Italian Association of Biomedical Physics 31 615-20

Granville D A and Sawakuchi G O 2015 Comparison of linear energy transfer scoring techniques in Monte Carlo simulations of proton beams Physics in medicine and biology $60 \mathrm{~N} 283-91$

Guan F, Peeler C, Bronk L, Geng C, Taleei R, Randeniya S, Ge S, Mirkovic D, Grosshans D, Mohan R and Titt U 2015 Analysis of the track- and dose-averaged LET and LET spectra in proton therapy using the geant 4 Monte Carlo code Medical physics $\mathbf{4 2}$ 6234-47

Guardiola C, Peucelle C and Prezado Y 2017 Optimization of the mechanical collimation for minibeam generation in proton minibeam radiation therapy Medical physics 44 14708

Hlatky L, Sachs R K, Vazquez M and Cornforth M N 2002 Radiation-induced chromosome aberrations: insights gained from biophysical modeling BioEssays : news and reviews in molecular, cellular and developmental biology 24 714-23

Hoshi M, Antoku S, Nakamura N, Russell W J, Miller R C, Sawada S, Mizuno M and Nishio S 1988 Soft X-ray dosimetry and RBE for survival of Chinese hamster V79 cells International journal of radiation biology 54 577-91

Ibahim M J, Crosbie J C, Yang Y, Zaitseva M, Stevenson A W, Rogers P A and Paiva P 2014 An evaluation of dose equivalence between synchrotron microbeam radiation therapy and conventional broad beam radiation using clonogenic and cell impedance assays PloS one 9 e 100547

Kaplan G I, Rosenfeld A B, Allen B J, Booth J T, Carolan M G and Holmes-Siedle A 2000 Improved spatial resolution by MOSFET dosimetry of an x-ray microbeam Medical physics 27 239-44

Kirkpatrick J P, Meyer J J and Marks L B 2008 The linear-quadratic model is inappropriate to model high dose per fraction effects in radiosurgery Semin Radiat Oncol 18 240-3

Klodowska M, Olko P and Waligorski M P R 2015 Proton microbeam radiotherapy with scanned pencil-beams - Monte Carlo simulations Phys Medica 31 621-6

Laissue J A, Geiser G, Spanne P O, Dilmanian F A, Gebbers J O, Geiser M, Wu X Y, Makar M S, Micca P L, Nawrocky M M, Joel D D and Slatkin D N 1998 Neuropathology of ablation of rat gliosarcomas and contiguous brain tissues using a microplanar beam of synchrotron-wiggler-generated X rays Int J Cancer 78 654-60

Lee E, Eagle J, Sandison G, Cao N, Stewart R, Marsh S and Meyer J 2016a SU-F-T-671: Effects of Collimator Material On Proton Minibeams Medical physics 433618

Lee E, Meyer J and Sandison G 2016b Collimator design for spatially-fractionated proton beams for radiobiology research Physics in medicine and biology 61 5378-89

Mairani A, Dokic I, Magro G, Tessonnier T, Kamp F, Carlson D J, Ciocca M, Cerutti F, Sala P R, Ferrari A, Bohlen T T, Jakel O, Parodi K, Debus J, Abdollahi A and Haberer T 2016 Biologically optimized helium ion plans: calculation approach and its in vitro validation Physics in medicine and biology 61 4283-99

Niemierko A 1997 Reporting and analyzing dose distributions: a concept of equivalent uniform dose Medical physics 24 103-10 
Nikjoo H and Lindborg L 2010 RBE of low energy electrons and photons Physics in medicine and biology 55 R65-109

Paganetti H 2014 Relative biological effectiveness (RBE) values for proton beam therapy. Variations as a function of biological endpoint, dose, and linear energy transfer Physics in medicine and biology 59 R419-72

Peng V, Suchowerska N, Rogers L, Mackonis E C, Oakes S and McKenzie D R 2017 Grid therapy using high definition multileaf collimators: realizing benefits of the bystander effect Acta Oncol 56 1048-59

Perl J, Shin J, Schumann J, Faddegon B and Paganetti H 2012 TOPAS: an innovative proton Monte Carlo platform for research and clinical applications Medical physics 396818 37

Peucelle C, Nauraye C, Patriarca A, Hierso E, Fournier-Bidoz N, Martinez-Rovira I and Prezado Y 2015 Proton minibeam radiation therapy: Experimental dosimetry evaluation Medical physics 42 7108-13

Polster L, Schuemann J, Rinaldi I, Burigo L, McNamara A L, Stewart R D, Attili A, Carlson D J, Sato T, Ramos Mendez J, Faddegon B, Perl J and Paganetti H 2015 Extension of TOPAS for the simulation of proton radiation effects considering molecular and cellular endpoints Physics in medicine and biology 60 5053-70

Prezado Y and Fois G R 2013 Proton-minibeam radiation therapy: a proof of concept Medical physics $\mathbf{4 0} 031712$

Ptaszkiewicz M, Braurer-Kirsch E, Klosowski M,Czopyk L and Olko P 2008 TLD dosimetry for microbeam radiation therapy at the European Synchrotron Radiation Facility Radiat Meas 43 990-3

Saini J, Maes D, Egan A, Bowen S R, St James S, Janson M, Wong T and Bloch C 2017 Dosimetric evaluation of a commercial proton spot scanning Monte-Carlo dose algorithm: comparisons against measurements and simulations Physics in medicine and biology 62 7659-81

Schmid T E, Greubel C, Hable V, Zlobinskaya O, Michalski D, Girst S, Siebenwirth C, Schmid E, Molls M, Multhoff G and Dollinger G 2012 Low LET protons focused to submicrometer shows enhanced radiobiological effectiveness Physics in Medicine \& Biology 575889

Serduc R, Christen T, Laissue J, Farion R, Bouchet A, Sanden B, Segebarth C, Brauer-Krisch E, Le Duc G, Bravin A, Remy C and Barbier E L 2008 Brain tumor vessel response to synchrotron microbeam radiation therapy: a short-term in vivo study Physics in medicine and biology 53 3609-22

Siegbahn E A, Stepanek J, Brauer-Krisch E and Bravin A 2006 Determination of dosimetrical quantities used in microbeam radiation therapy (MRT) with Monte Carlo simulations Medical physics 33 3248-59

Slatkin D N, Spanne P, Dilmanian F A and Sandborg M 1992 Microbeam radiation therapy Medical physics 19 1395-400

Spadinger I and Palcic B 1992 The relative biological effectiveness of 60Co gamma-rays, 55 $\mathrm{kVp}$ X-rays, $250 \mathrm{kVp}$ X-rays, and $11 \mathrm{MeV}$ electrons at low doses International journal of radiation biology $61345-53$

Stewart R D, Streitmatter S W, Argento D C, Kirkby C, Goorley J T, Moffitt G, Jevremovic T and Sandison G A 2015 Rapid MCNP simulation of DNA double strand break (DSB) relative biological effectiveness (RBE) for photons, neutrons, and light ions Physics in medicine and biology 60 8249-74

Stewart R D, Yu V K, Georgakilas A G, Koumenis C, Park J H and Carlson D J 2011 Effects of radiation quality and oxygen on clustered DNA lesions and cell death Radiation research $176587-602$ 
Streitmatter S W, Stewart R D, Jenkins P A and Jevremovic T 2017 DNA double strand break (DSB) induction and cell survival in iodine-enhanced computed tomography (CT) Physics in medicine and biology 62 6164-84

Zeman W, Curtis H J and Baker C P 1961 Histopathologic effect of high-energy-particle microbeams on the visual cortex of the mouse brain Radiation research 15 496-514

Zeman W, Curtis H J, Gebhard E L and Haymaker W 1959 Tolerance of mouse-brain tissue to high-energy deuterons Science 130 1760-1

Zhang H L, Zhong H L, Barth R F, Cao M S and Das I J 2014a Impact of dose size in single fraction spatially fractionated (grid) radiotherapy for melanoma Medical Physics $\mathbf{4 1}$

Zhang L, Yuan H, Burk L M, Inscoe C R, Hadsell M J, Chtcheprov P, Lee Y Z, Lu J, Chang $\mathrm{S}$ and Zhou O 2014b Image-guided microbeam irradiation to brain tumour bearing mice using a carbon nanotube $\mathrm{x}$-ray source array Physics in medicine and biology $\mathbf{5 9}$ 1283-303

Zlobinskaya O, Girst S, Greubel C, Hable V, Siebenwirth C, Walsh D W, Multhoff G, Wilkens J J, Schmid T E and Dollinger G 2013 Reduced side effects by proton microchannel radiotherapy: study in a human skin model Radiation and environmental biophysics 52 123-33 


\section{Figures}

2

5

6

7

8

9

10

11

12

13

14

15

16

17

18

19

20

21

22

23

24

25

26

27

28

29

30

31

32

33

34

35

36

37

38

39

40

41

42

43

44

45

46

47

48

49

50

51

52

53

54

55

56

57

58

59

60

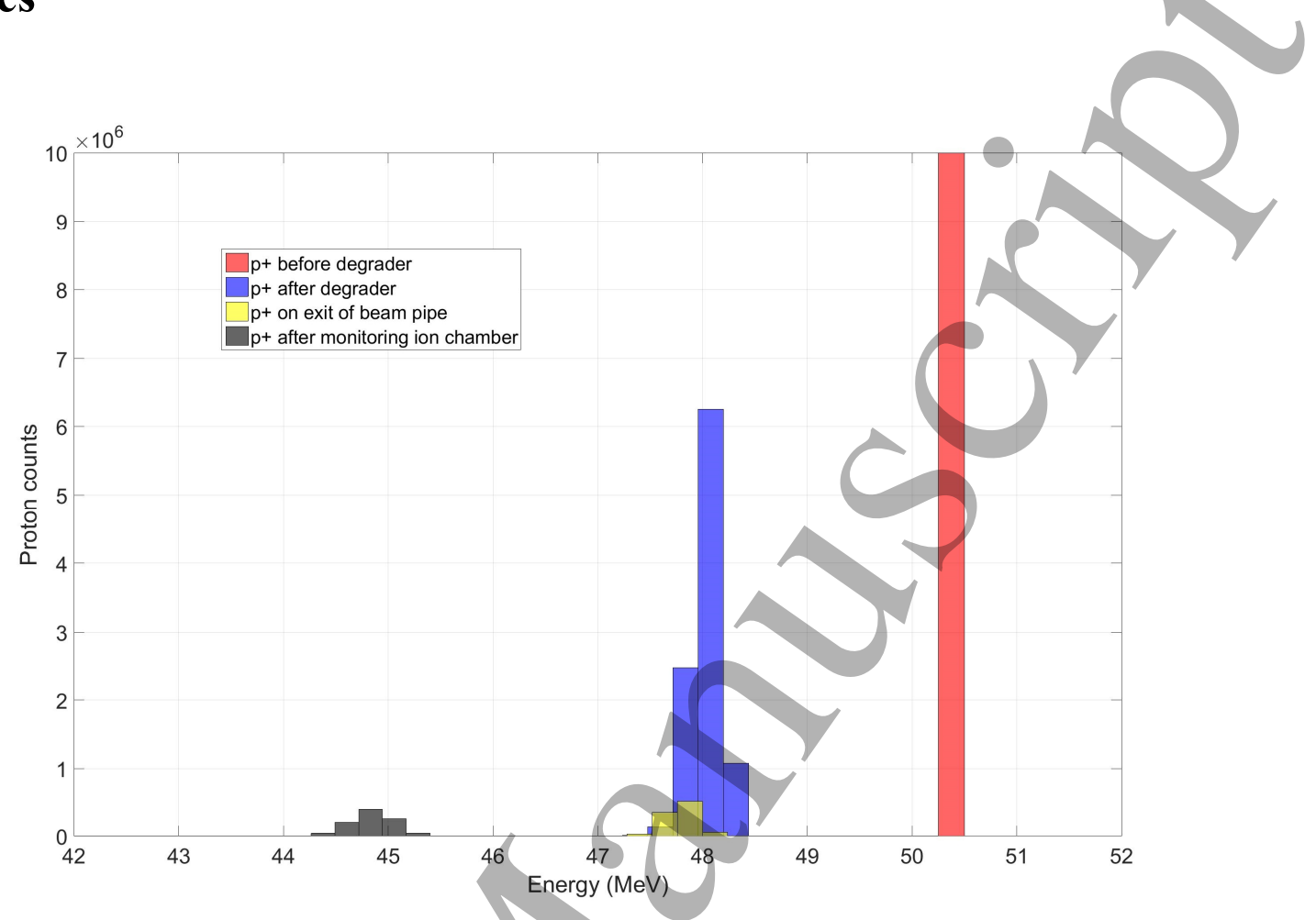

Fig. 1: Analysis of the effects of beam components on the proton energy spectrum for the open $3.5 \mathrm{~cm}$ diameter $50.5 \mathrm{MeV}$ proton beam from the UW cyclotron. Each energy bin is $0.25 \mathrm{MeV}$. 


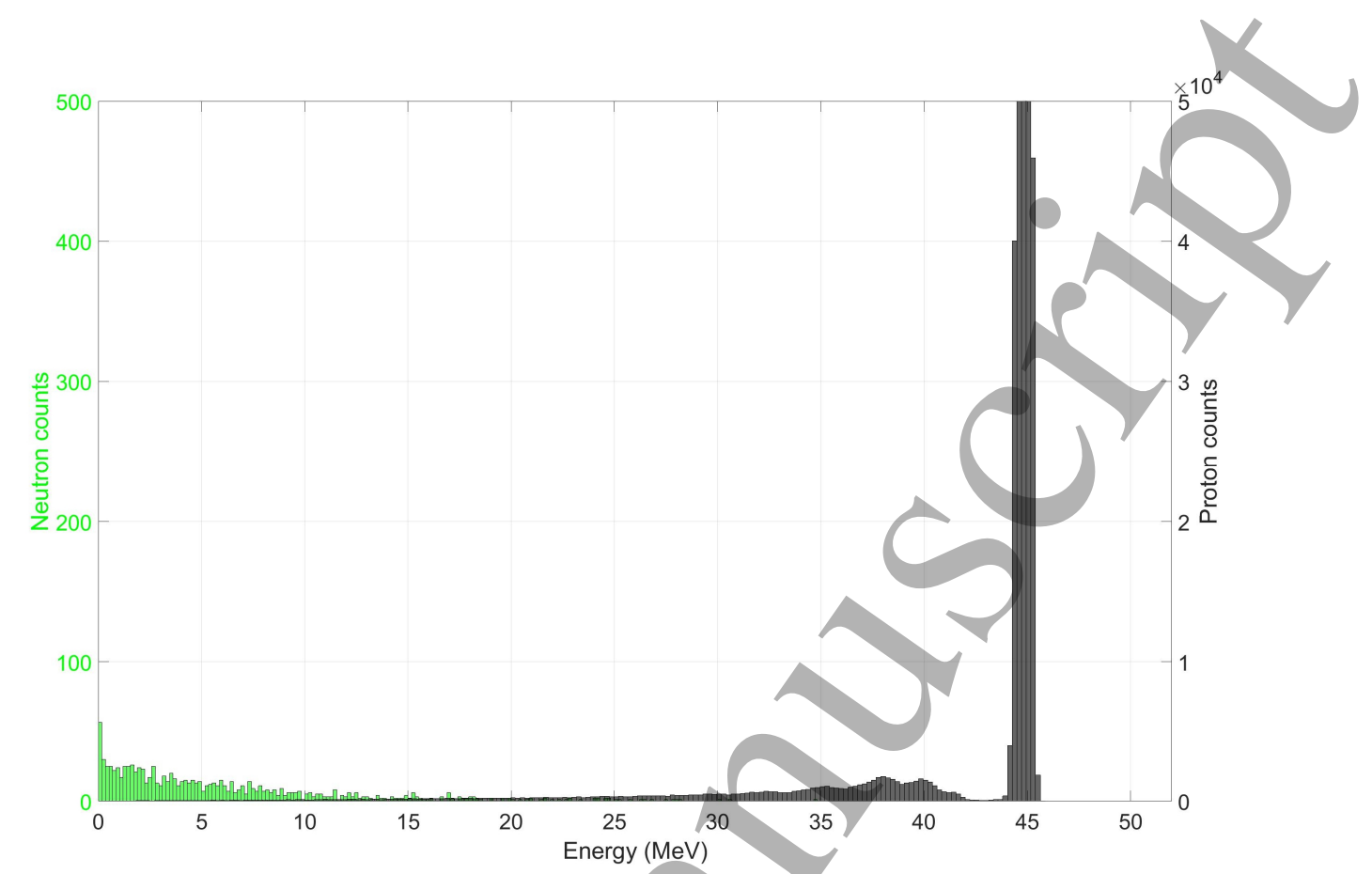

(a)

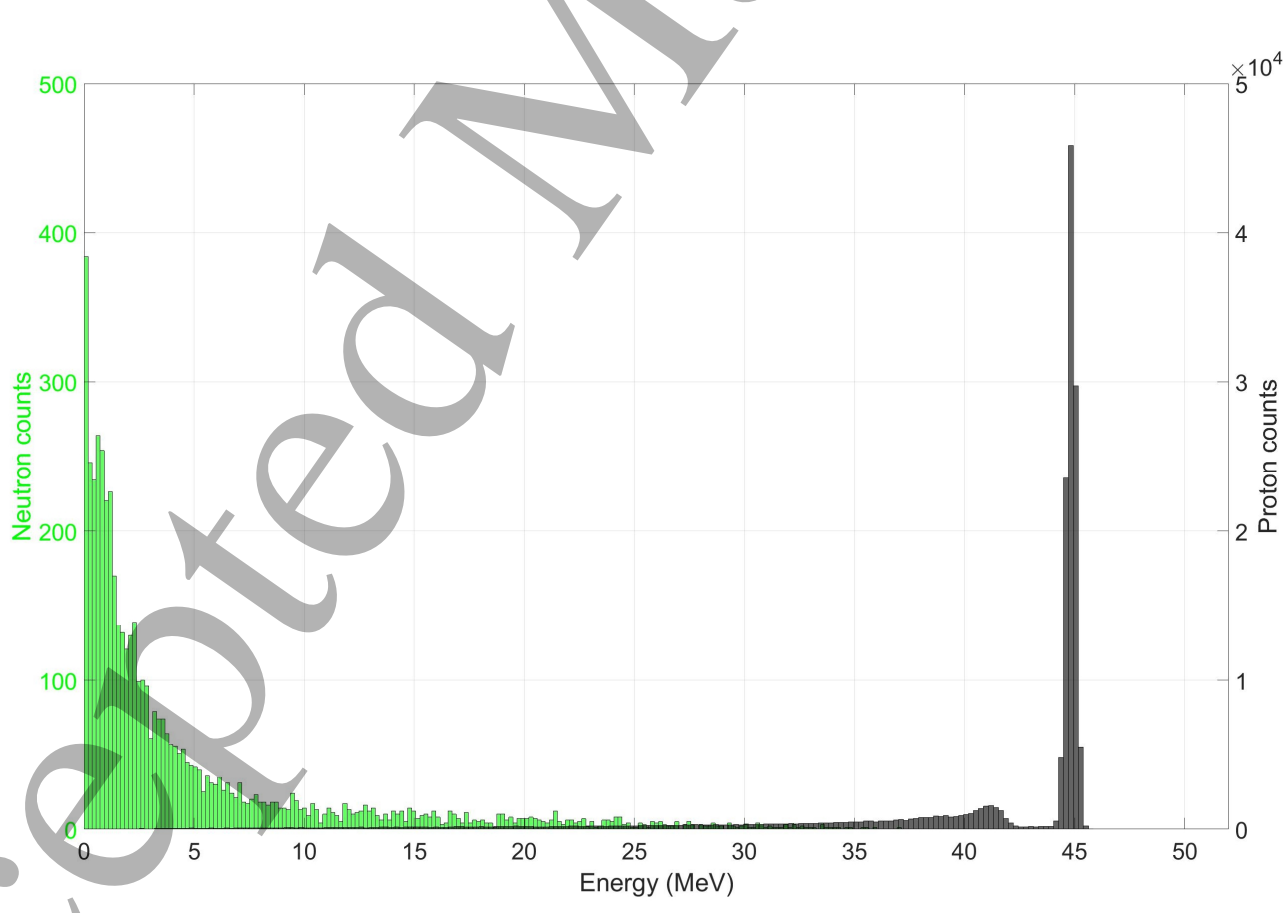

(b) 
Meyer et al

1
2
3
4
5
6
7
8
9
10
11
12
13
14
15
16
17
18
19
20
21
22
23
24
25
26
27
28
29
30
31
32
33
34
35
36
37
38
39
40
48
49
50
50
51
52
53
54
55
50

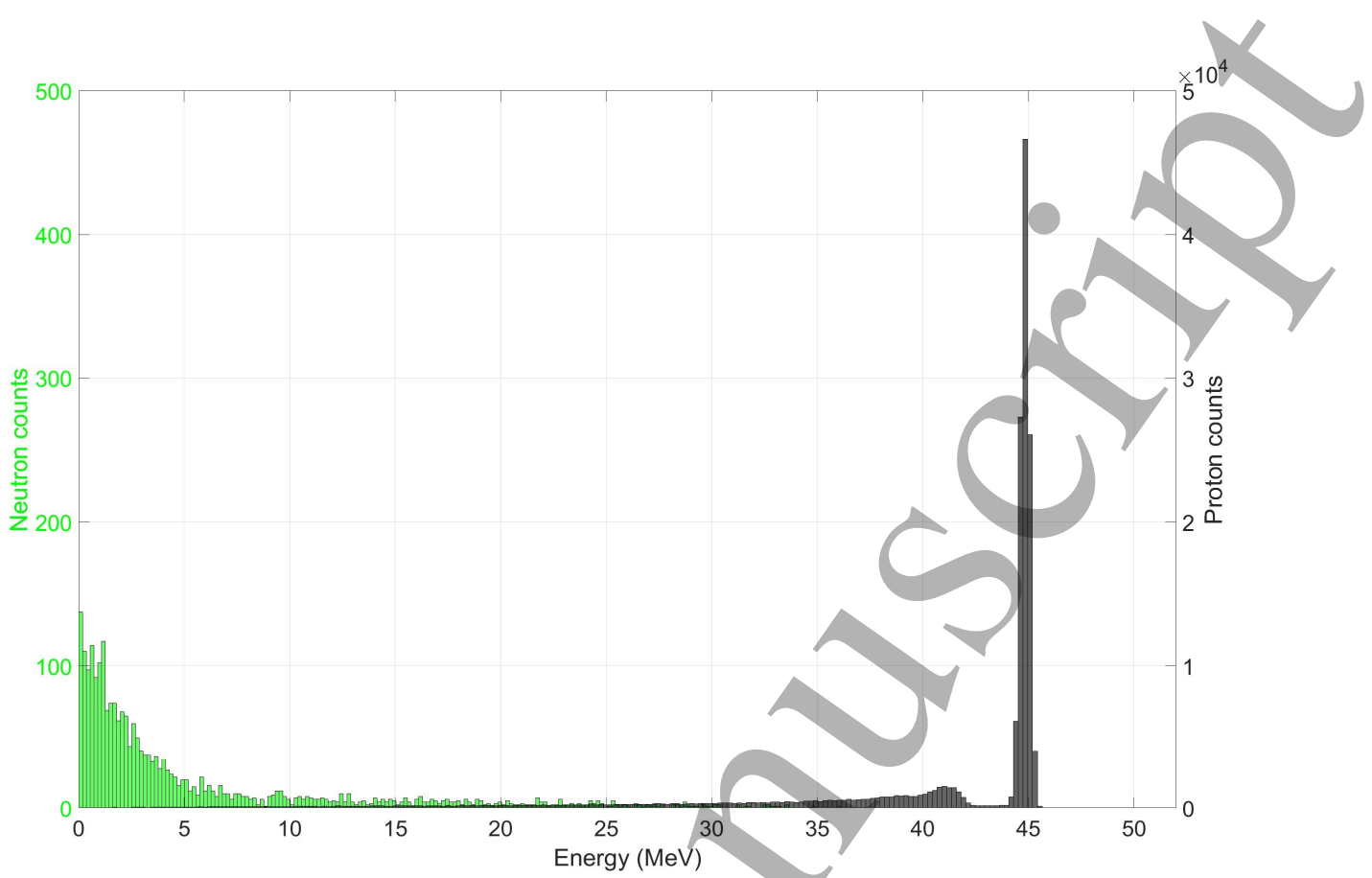

(c)

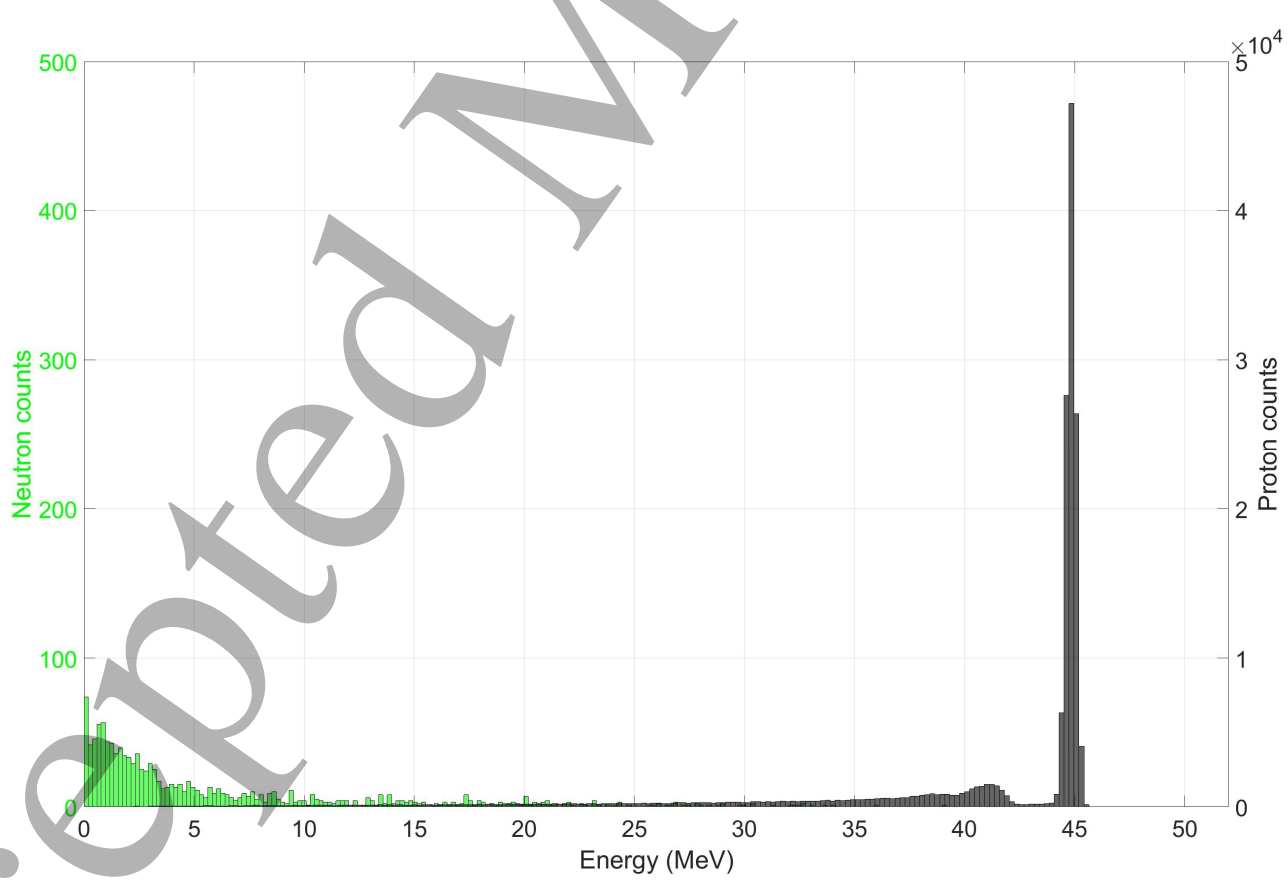

(d) 
1

2

3

4

5

6

7

8

9

10

11

12

13

14

15

16

17

18

19

20

21

22

23

24

25

26

27

28

29

30

31

32

33

34

35

36

37

38

39

40

41

42

43

44

45

46

47

48

49

50

51

52

53

54

55

56

57

58

59

60

Fig. 2: Proton (black) and neutron (green) energy spectra at entrance of water phantom with a) no collimator, b) collimator placed flush at water phantom, c) collimator placed at $2 \mathrm{~cm}$ distance and d) collimator placed at $5 \mathrm{~cm}$ distance from the water phantom. Left axis shows relative neutron counts and right axis relative proton counts. Note that the proton counts for the open beam in a), corresponding to $p+$ after monitoring ion chamber in Fig. 1, are truncated to be on the same scale as the collimated beam (Figs. 2b-d).

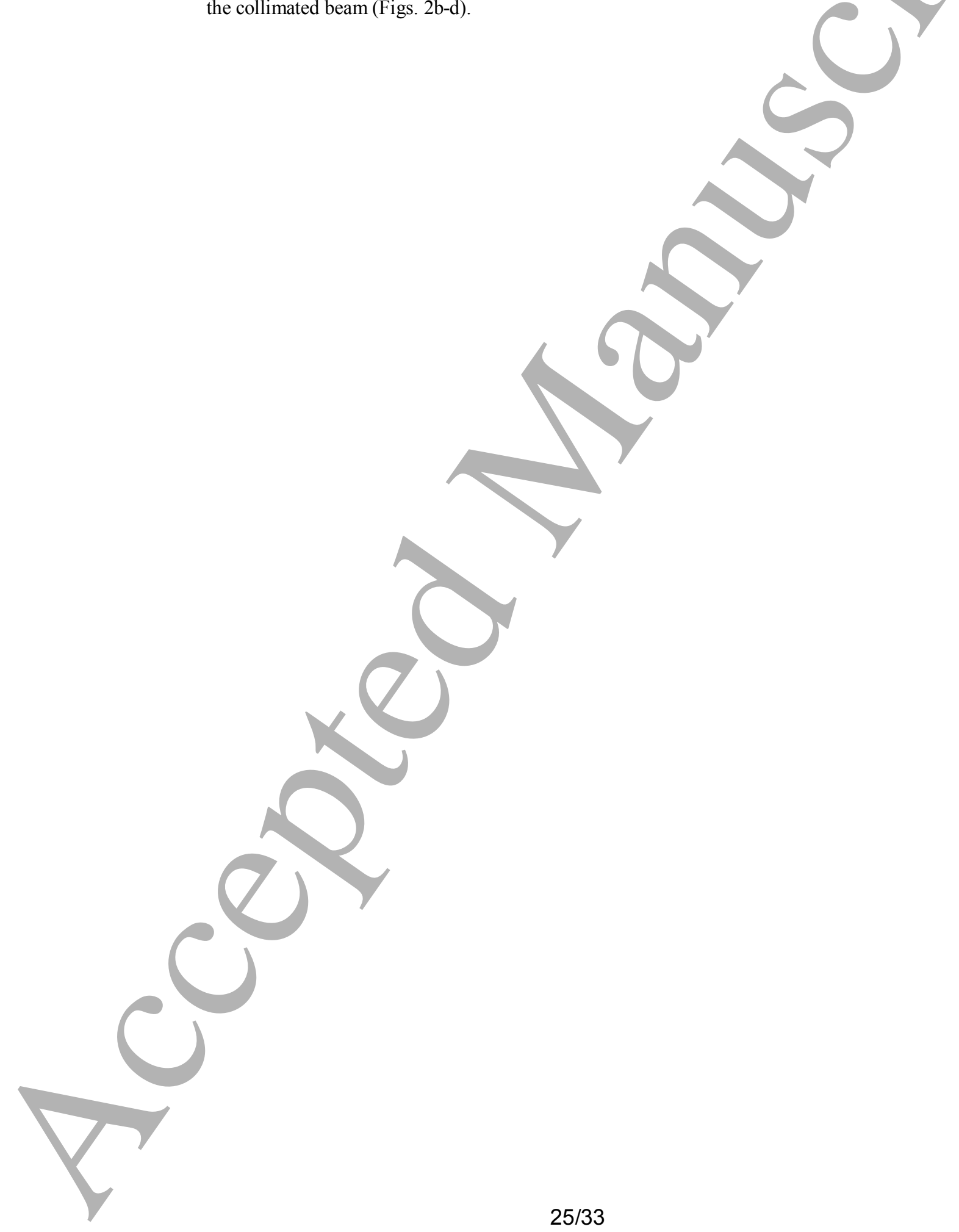




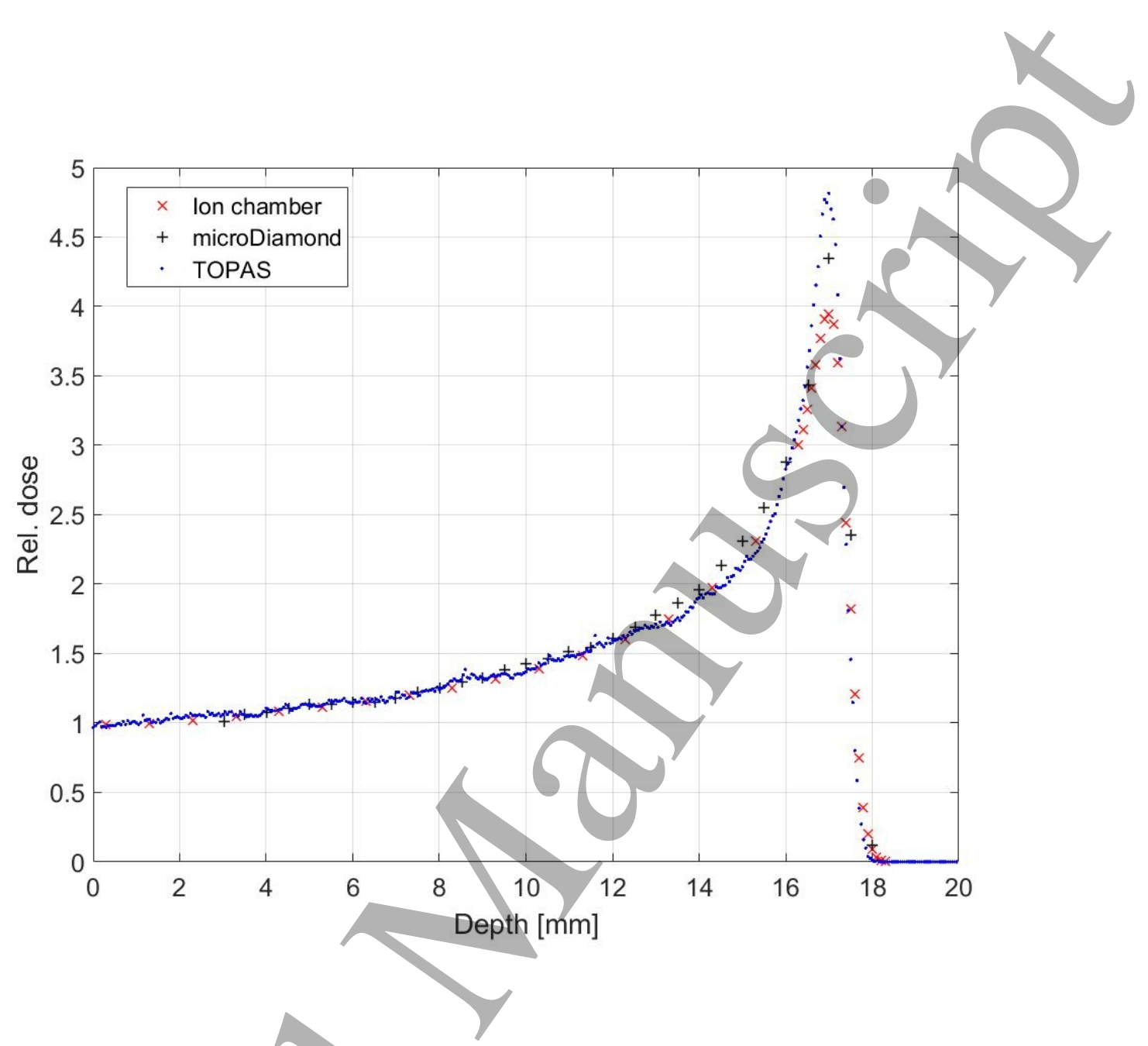

Fig. 3: Comparison of the measured vs modelled PDDs for the open field. 

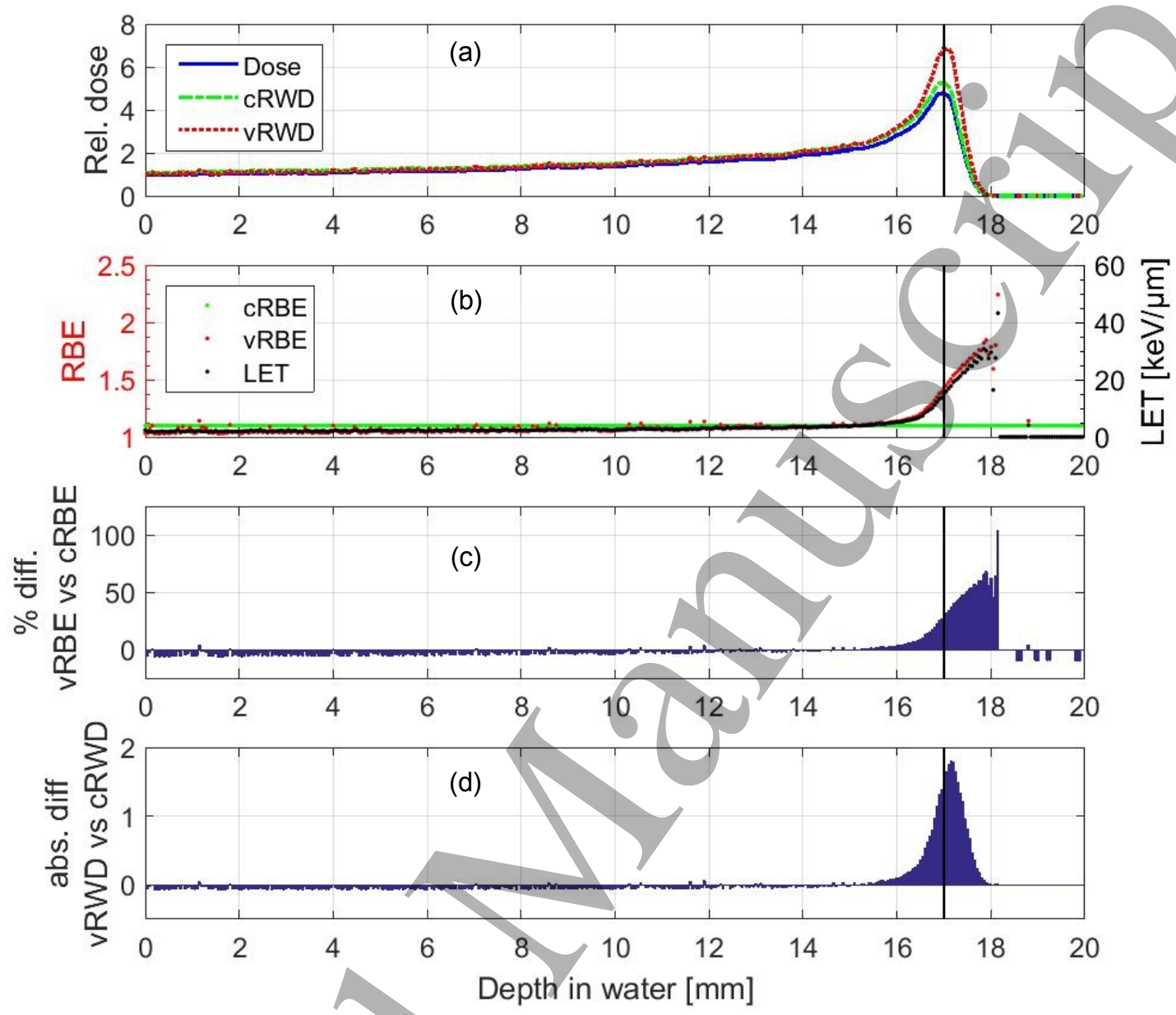

Fig. 4: Percentage depth dose comparison of physical vs biological dose. a) Physical dose (dose) vs RWD based on a constant RBE of 1.1 (cRWD) vs RWD based on variable RBEDSB (vRWD), b) cRBE and $\mathrm{RBE}_{\mathrm{DSB}}(\mathrm{vRBE})$ as a function of depth (primary axis on left) plus LET as a function of depth (secondary axis on right), c) percentage difference between $\mathrm{RBE}_{\mathrm{DSB}}$ and cRBE with depth, d) absolute difference between cRWD and vRWD with depth. 

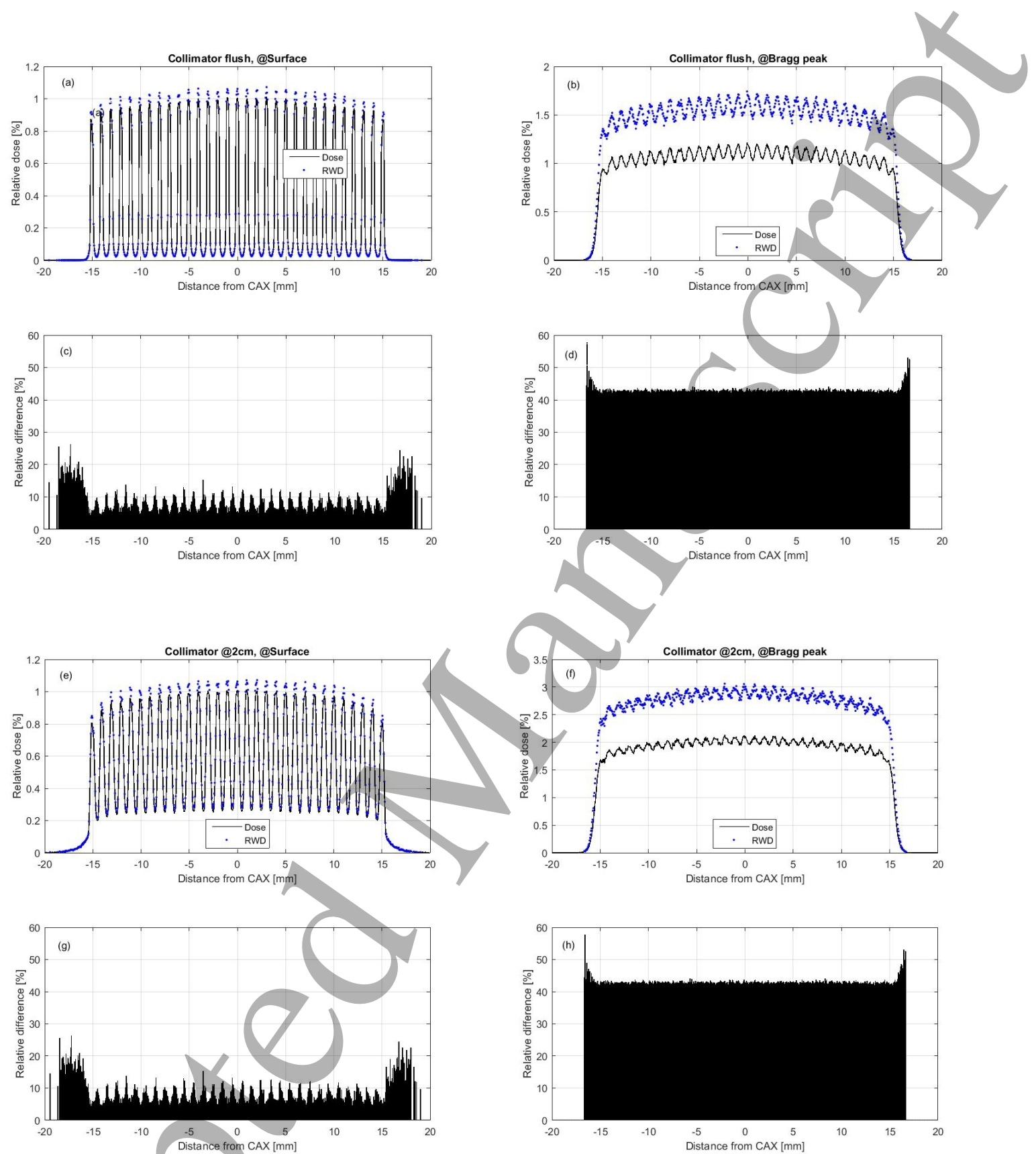

Fig. 5: Dose profiles of physical dose vs RWD normalized to dose at central axis at $1 \mathrm{~mm}$ depth. a) at surface for collimatorl flush, b) at BP for collimator flush, c) relative difference at surface for collimator flush, d) relative difference at BP for collimator flush, e) at surface for collimator at $2 \mathrm{~cm}$ distant, f) at Bragg peak for collimator at $2 \mathrm{~cm}$ distant, c) relative difference at surface for collimator at $2 \mathrm{~cm}$ distant, $\mathrm{d}$ ) relative difference at $\mathrm{BP}$ for collimator at $2 \mathrm{~cm}$ distant. 

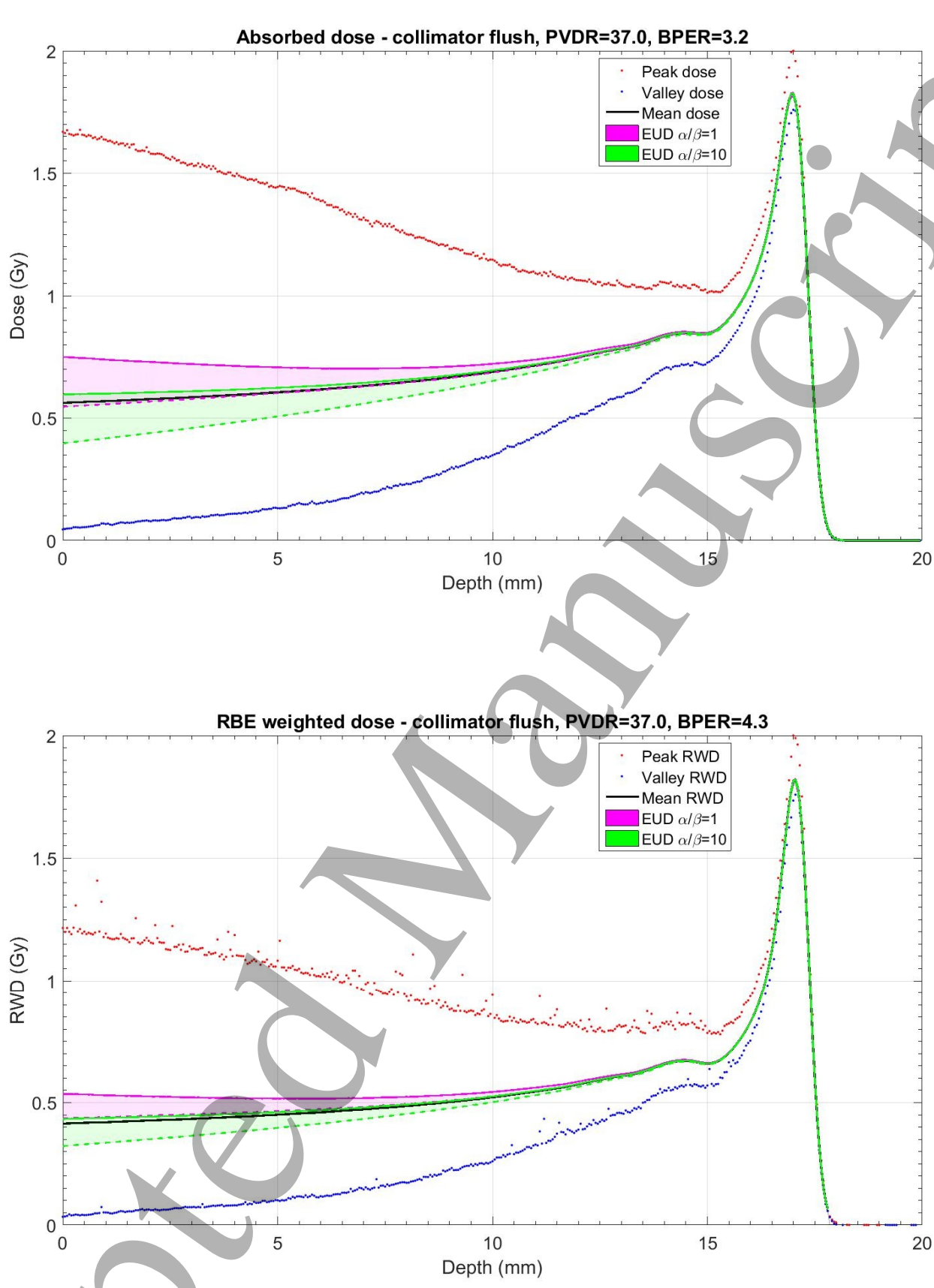

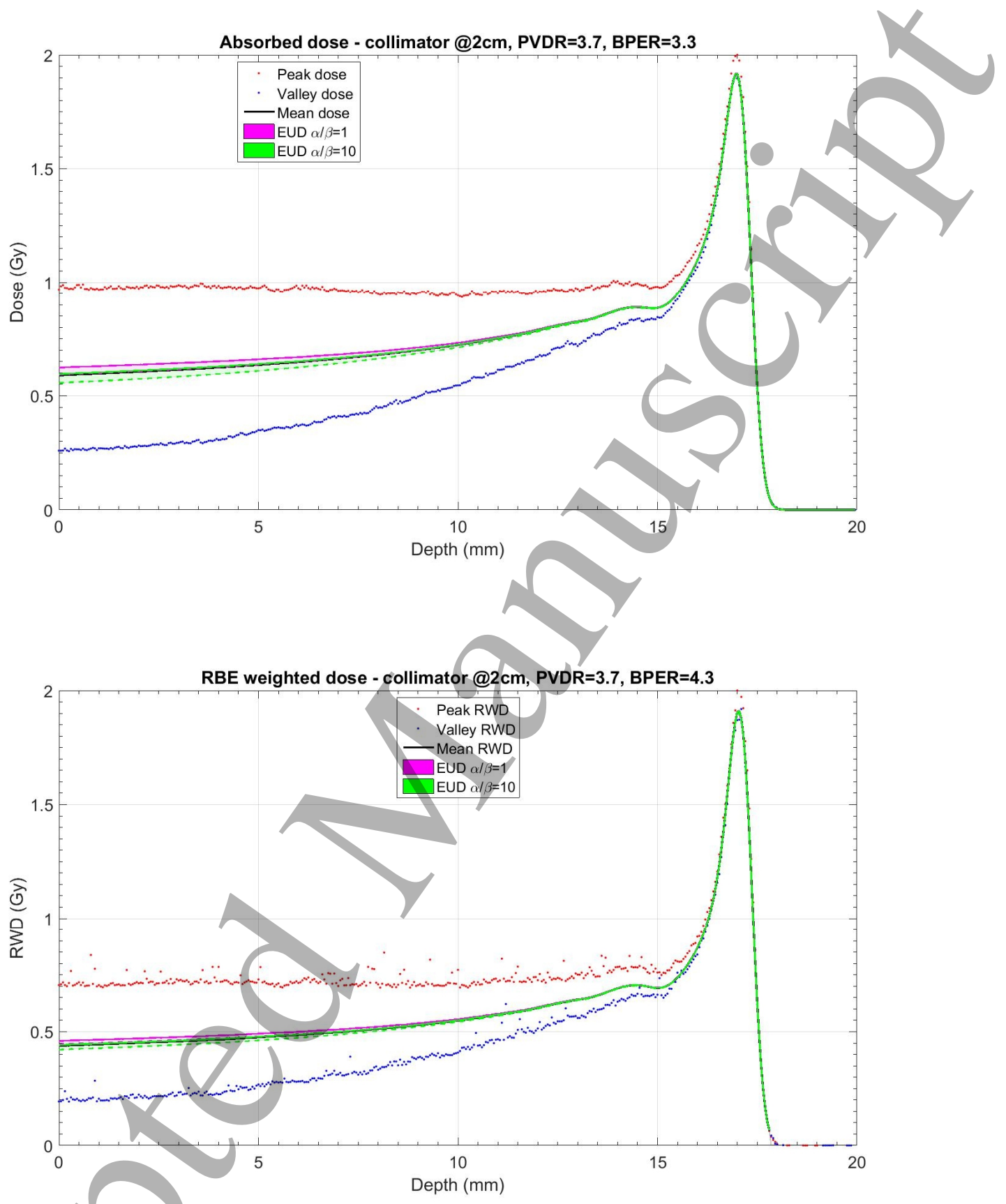


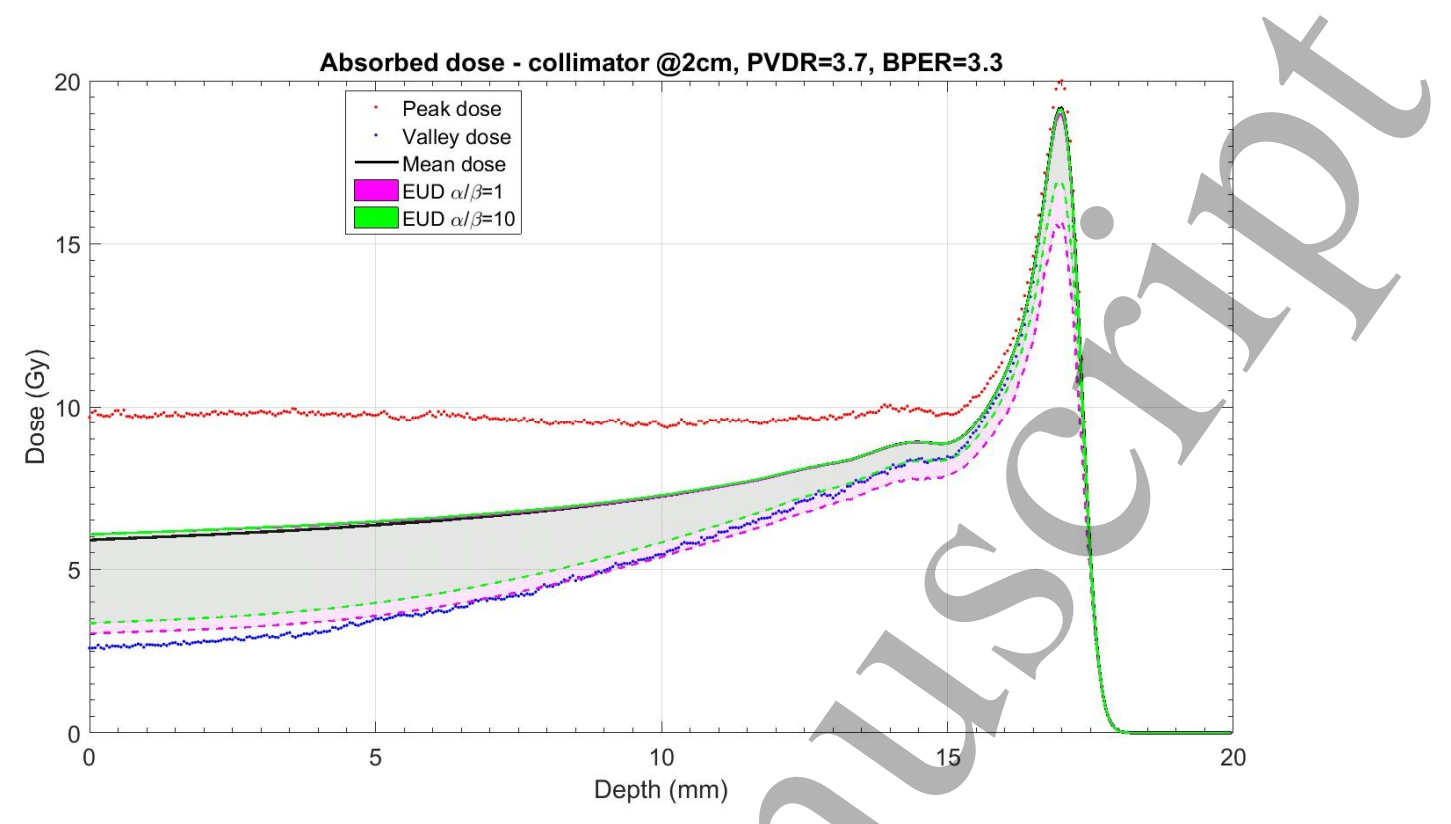

Fig. 6: Comparison of mean dose versus EUD for absorbed dose along CAX (a, c, e) and RWD (b, d, f) for a range of $\boldsymbol{\alpha} / \boldsymbol{\beta}$ and $\overline{\mathbf{S}}=0.1$ to 0.95 for proton minibeams. The dashed EUD lines correspond to $\overline{\mathbf{S}}=0.1$ (radiosensitive) and the solid lines to $\overline{\mathbf{S}}=0.95$ (radioresistant). a) 2 Gy dose with collimator flush b) 2

Gy dose with collimator at $2 \mathrm{~cm}$ distance, c) RWD corresponding to a), d) RWD corresponding to b), e) 20 Gy dose with collimator at $2 \mathrm{~cm}$ distance, f) RWD corresponding to e). PVDR: Peak-tovalley-dose-ratio, BPER: Bragg-peak-to-entrance-dose-ratio. 
Meyer et al

\section{Tables}

Table 1: $L E T$ and $R_{B E}$ DSB values for a range of beam energies for an open proton beam on the UW beamline.

\begin{tabular}{|c|c|c|c|c|c|c|c|c|c|c|c|}
\hline \multicolumn{2}{|c|}{ nominal beam energy (MeV) } & \multicolumn{2}{|c|}{30} & \multicolumn{2}{|c|}{50.5} & \multicolumn{2}{|c|}{62} & \multicolumn{2}{|c|}{100} & \multicolumn{2}{|c|}{109} \\
\hline \multicolumn{2}{|c|}{ depth BP $(\mathrm{mm})$} & \multicolumn{2}{|c|}{3.2} & \multicolumn{2}{|c|}{17.0} & \multicolumn{2}{|c|}{27.0} & \multicolumn{2}{|c|}{71.3} & \multicolumn{2}{|c|}{84.0} \\
\hline & & RBE & $\begin{array}{c}\text { LET } \\
(\mathrm{keV} / \mu \mathrm{m})\end{array}$ & RBE & $\begin{array}{c}\text { LET } \\
(\mathrm{keV} / \mu \mathrm{m})\end{array}$ & RBE & $\begin{array}{c}\text { LET } \\
(\mathrm{keV} / \mu \mathrm{m})\end{array}$ & RBE & $\begin{array}{c}\text { LET/ } \\
(\mathrm{keV} / \mu \mathrm{m})\end{array}$ & & $\begin{array}{c}\text { LET } \\
(\mathrm{keV} / \mu \mathrm{m})\end{array}$ \\
\hline \multirow[t]{2}{*}{ location } & $\mathrm{BP}$ & 1.57 & 20.00 & 1.43 & 15.13 & 1.34 & 12.06 & 1.28 & 10.02 & 1.26 & 9.65 \\
\hline & distal end & 2.02 & 38.07 & 1.85 & 30.09 & 1.73 & 28.40 & 1.49 & 18.05 & 1.38 & 13.58 \\
\hline
\end{tabular}


1

2

3

4

5

6

7

8

9

10

11

12

13

14

15

16

17

18

19

20

21

22

23

24

25

26

27

28

29

30

31

32

33

34

35

36

37

38

39

40

41

42

43

44

45

46

47

48

49

50

51

52

53

54

55

56

57

58

59

60

Table 2: $\mathrm{LET}$ and $\mathrm{RBE}_{\mathrm{DSB}}$ values at the BP with and without collimator for UW beamline with $50.5 \mathrm{MeV}$ nominal beam energy.

\begin{tabular}{ccccc}
\hline & open & \multicolumn{3}{c}{ collimator } \\
\hline $\begin{array}{c}\text { collim. distance to } \\
\text { phantom }(\mathrm{cm})\end{array}$ & $\begin{array}{c}\text { not } \\
\text { applicable }\end{array}$ & 0 & 2 & 5 \\
\hline LET @BP & 15.1 & 16.3 & 15.4 & 14.6 \\
\hline RBE @BP & 1.4 & 1.5 & 1.4 & 1.4 \\
\hline
\end{tabular}

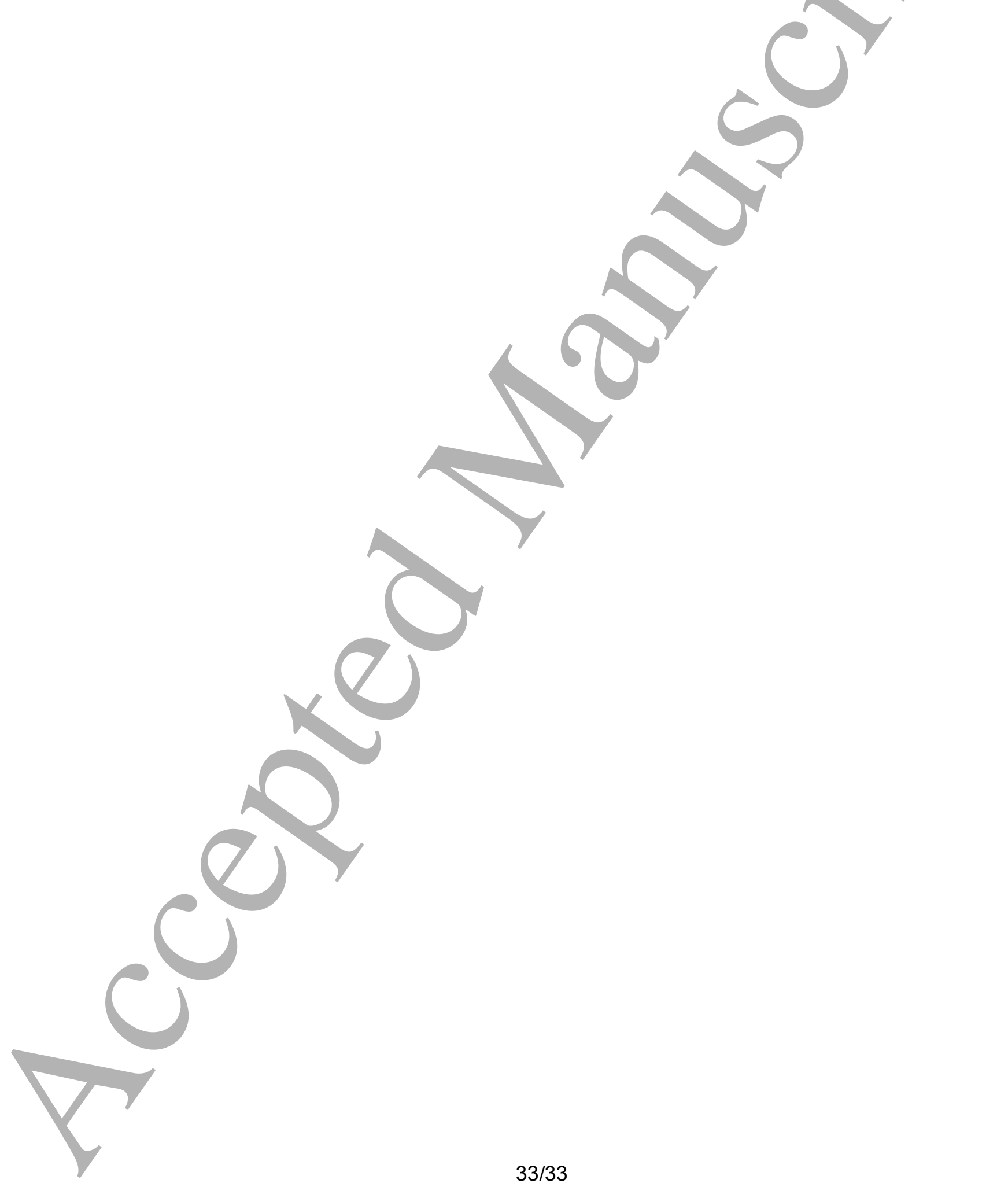

